\title{
Pricing Intertemporal Risk When Investment Opportunities Are Unobservable
}

\author{
Scott Cederburg*
}

\begin{abstract}
The intertemporal capital asset pricing model (ICAPM) predicts that an unobservable factor capturing changes in expected market returns should be priced in the cross section. My Bayesian framework accounts for uncertainty in the intertemporal risk factor and gauges the effects of prior information about investment opportunities on model inferences. Whereas an uninformative prior specification produces weak evidence that intertemporal risk is priced, incorporating prior information about market-return predictability generates a large space of ex ante reasonable priors in which the estimated intertemporal risk factor is positively priced. Overall, the cross-sectional tests reject the capital asset pricing model (CAPM) and indicate support for the ICAPM.
\end{abstract}

\section{Introduction}

The intertemporal capital asset pricing model (ICAPM) of Merton (1973) and Campbell (1993) predicts that factors that capture unexpected shifts in the investment opportunity set should be priced in the cross section. The capital asset pricing model (CAPM) is likely to be inadequate for explaining the cross section of expected returns under this framework, and the ICAPM frequently serves as theoretical motivation for additional factors in empirical multifactor models. Importantly, Campbell's (1993) ICAPM identifies a specific additional factor that is generated by time variation in expected market returns. As such, the ICAPM is

*Cederburg (corresponding author), cederburg@email.arizona.edu, University of Arizona Eller College of Management. I thank David Bates, Matt Billett, David Brown, Jennifer Conrad (the editor), Phil Davies, Robert Dittmar, Redouane Elkamhi, Massimo Guidolin (the referee), Huseyin Gulen, Arthur Korteweg, Chris Lamoureux, Mike O’Doherty, B. Ravikumar, Rick Sias, Ashish Tiwari, Paul Weller, and Yu Yuan; seminar participants at the University of Arizona, the University of Connecticut, Georgia Tech, Indiana University, the University of Missouri, Purdue University, Rutgers University, and the University of Texas at Dallas; and participants at the Financial Management Association Doctoral Student Consortium, the 2010 Center for Research in Security Prices Forum, the 2011 Eastern Finance Association Conference, and the 2011 Midwest Finance Association Conference for helpful comments and suggestions. An allocation of computer time from High Performance Computing (HPC) at the University of Arizona is gratefully acknowledged. Any errors are my own. 
an important theory for understanding asset prices because it ties together timeseries and cross-sectional aspects of returns (Fama (1991)).

A primary difficulty in testing the ICAPM of Campbell (1993) is that its implied intertemporal risk factor, which captures unanticipated changes in expected future market returns, is unobservable. Furthermore, the return-predictability literature demonstrates that market-return forecasts are marked by considerable uncertainty (e.g., Kandel and Stambaugh (1996), Barberis (2000)). Empirical implementations of Campbell's (1993) ICAPM estimate the intertemporal risk factor using market-return forecasts, so uncertainty in the latent factor is an important consideration in tests of the model.

In this article, I adopt a Bayesian approach that is well suited to test the cross-sectional implications of the ICAPM given the unobservability of the intertemporal risk factor. I specifically examine whether exposure to intertemporal risk is priced in the cross section of stocks using tests that consider the full posterior distribution of the factor time series, such that the resulting prices of risk reflect uncertainty in the estimated factor. In addition, I explore the effects of prior information about market-return dynamics on estimates of the intertemporal risk factor and the subsequent ICAPM tests. Informative priors are potentially important in this setting given that the data are relatively uninformative about the return-predictability relation.

Prior information about investment opportunities is available from several potential sources. As an important example, it may be expected ex ante that realizations of the intertemporal risk factor of Campbell (1993) will be negatively correlated with market returns. In particular, the intertemporal risk factor corresponds to the discount-rate component in the Campbell (1991) decomposition of returns into the components of discount-rate and cash-flow news. Positive shocks to discount rates have a negative influence on current market returns, and the implied negative relation between the intertemporal risk factor and the market factor is important for ICAPM inferences. In the extreme, if the intertemporal risk factor is perfectly negatively correlated with the market factor, then the CAPM beta of each firm is an adequate summary of risk exposure (Campbell (1993)). Alternatively, if market returns also reflect news about cash-flow expectations, then the two ICAPM factors may each produce a substantial incremental impact on expected returns.

I use the predictive-system approach of Pástor and Stambaugh (2009) to model the market risk premium and the risk-free rate, and the estimation procedure generates the posterior distribution of the time series for the intertemporal risk factor. The predictive-system approach has two advantages that are particularly useful in this context. First, whereas a predictive regression assumes that the market risk premium is an exact linear function of observed state variables, a predictive system allows for imperfect prediction and better reflects uncertainty in return forecasts. Second, the predictive-system approach allows me to directly place priors on economically interesting aspects of the return-predictability relation. Specifically, in some tests I specify informative prior beliefs about the $R^{2}$ from a predictive regression for market excess returns as well as the correlation between shocks to current returns and the market risk premium. 
I begin by testing the ICAPM over the 1952-2014 period with an intertemporal risk factor that is estimated from the predictive system with uninformative priors. In this specification, the intertemporal risk factor is only modestly correlated with the market factor, with a posterior average correlation of -0.23 , such that the additional factor may contain important incremental information about expected returns. Given the posterior of the intertemporal risk factor, I test the ICAPM using the full cross section of individual stocks. The results show that the market factor is positively priced in the cross section with a posterior mean of $0.63 \%$ per month (90\% credible interval of $0.25 \%-1.01 \%$ ), and the $90 \%$ credible interval of the intercept includes $0 .{ }^{1}$ The intertemporal risk factor has a positive price-of-risk posterior mean of $0.52 \%$ per month in the base specification, but the price of risk has a large posterior standard deviation of $0.44 \%$, and the $90 \%$ credible interval is $-0.11 \%$ to $1.30 \%$. Much of the uncertainty in the price of intertemporal risk is inherited from uncertainty about the latent factor; the posterior standard deviation of intertemporal risk factor realizations for a given month is $3.48 \%$ on average. Overall, the results for the intercept and market factor are in line with ICAPM predictions, but there is only weak evidence that intertemporal risk is priced in the cross section given uninformative priors.

I next consider the effects of prior information about the dynamics of the market risk premium, which can inform and potentially sharpen estimates of the intertemporal risk factor. To systematically study the impact of informative priors, I form a 2-dimensional space based on the predictive-system prior parameters that are particularly important for ICAPM inferences. The first dimension is a prior parameter for the $R^{2}$ in a predictive regression of monthly excess market returns on the market risk premium, and I consider a range of $0 \%-3 \%$ for this prior. The second dimension is the correlation between shocks to current returns and the market risk premium, $\rho_{m \bar{m}}$, and the priors have means ranging from -0.9 to 0.0 to place greater focus on $\rho_{m \bar{m}}<0$, as suggested by the Campbell (1991) decomposition. These two prior parameters are particularly interesting because they inform parameters that are i) relatively difficult to learn about from the data and ii) important for determining the relation between the two factors in the ICAPM.

To better understand the prior-parameter space and gauge which sets of priors are ex ante most sensible, I use a prior predictive analysis with stock return variance ratios. The $k$-year variance ratio is defined following Poterba and Summers (1988) as the variance of $k$-year cumulative returns divided by $k$ times the variance of 1-year returns. I show analytically that the $k$-year variance ratio is a function of the $R^{2}$ and $\rho_{m \bar{m}}$ parameters that define the grid space, with opposing effects from mean reversion and uncertainty about future expected returns. Over a long 1802-1951 period that predates the ICAPM testing period, historical variance ratios reach a low of 0.58 at an 8-year horizon, indicating substantial mean reversion in returns. After generating the prior distributions of 2- to 8-year variance ratios from the predictive system given each set of prior parameters, I demonstrate that priors associated with higher return predictability and a relatively large negative

\footnotetext{
${ }^{1}$ I calculate the $90 \%$ credible interval for a given parameter as the range that contains $90 \%$ of the posterior probability mass with equal $5 \%$ tails.
} 
correlation between shocks to current returns and the market risk premium are most consistent with the estimated variance ratios from historical stock returns.

I test the ICAPM using the estimated intertemporal risk factor associated with each set of prior parameters. The results for the price of market risk and intercept are similar to the specification with uninformative priors, such that ICAPM predictions are supported for these two parameters. More importantly, inferences about the pricing of intertemporal risk vary across the priors. Whereas many of the prior specifications produce little evidence that intertemporal risk is priced in the cross section, there is a relatively large region of the prior-parameter space in which exposure to intertemporal risk receives a large, positive reward. As a specific example, the prior specification with $R^{2}=2 \%$ and a prior mean of -0.45 for $\rho_{m \bar{m}}$ produces a posterior of the price of intertemporal risk that is centered at $0.97 \%$ per month, and the $90 \%$ credible interval is located well above 0 . Importantly, the region of the prior-parameter space in which intertemporal risk is positively priced has substantial overlap with the region that is deemed to be ex ante reasonable based on the prior predictive analysis.

Across the specifications with reasonable priors and positive ICAPM results, the estimated intertemporal risk factors share important characteristics. First, the posteriors of the predictive-regression $R^{2}$ indicate substantial market-return predictability, with posterior means in the range of $2.4 \%-2.8 \%$. Second, shocks to current returns and the market risk premium are moderately negatively correlated with posterior means of $\rho_{m \bar{m}}$ of approximately -0.4 . Third, the risk-free-rate and market-risk-premium components of the intertemporal risk factor are negatively correlated, such that increases in the real risk-free rate tend to occur contemporaneously with decreases in the risk premium. The negative relation between these shocks tends to smooth the intertemporal risk factor relative to alternative specifications. These aspects of return dynamics combine to produce intertemporal risk factors that have a moderately negative correlation with the market factor of approximately -0.3 to -0.4 . In the cases with positive ICAPM results, the predictive system thus indicates that news about both cash flows and discount rates plays an important role in stock returns.

As previously noted, the ICAPM is important for understanding asset prices in this context because it ties together the time-series and cross-sectional aspects of returns. The cross-sectional tests in my article provide evidence of an additional priced risk factor in the cross section of stocks, which constitutes a rejection of the CAPM. Under specific sets of ex ante reasonable priors about market-return predictability, this additional factor is also consistent with time-series properties of returns and corresponds to the intertemporal risk factor in Campbell's (1993) ICAPM. My findings thus provide empirical support for the theoretical prediction of the ICAPM that intertemporal risk is priced in the cross section of stocks.

This article contributes to the literature that tests the cross-sectional implications of the ICAPM. Several studies test the ICAPM using estimated intertemporal risk factors that are disciplined to be consistent with time-series aspects of returns (e.g., Campbell (1996), Hodrick, Ng, and Sengmueller (1999), Brennan, Wang, and Xia (2004), Campbell and Vuolteenaho (2004), Campbell, Polk, and Vuolteenaho (2010), Campbell, Giglio, and Polk (2013), Maio (2013), and Campbell, Giglio, Polk, and Turley (2018)). Whereas the bulk of previous 
research tests the ICAPM using portfolios, my article and that of Boons (2016) test the ICAPM among individual stocks to avoid potential problems with portfolio formation. I make contributions to the literature by explicitly accounting for uncertainty in the latent intertemporal risk factor and investigating the role of prior information about investment opportunities in inferences about intertemporal risk.

This article is also related to the literature that investigates uncertainty and informative priors in asset pricing. Kandel and Stambaugh (1996), Avramov (2002), (2004), Cremers (2002), Avramov and Chordia (2006), Pástor and Stambaugh (2009), (2012), Wachter and Warusawitharana (2009), (2015), Pettenuzzo, Timmermann, and Valkanov (2014), and Avramov, Cederburg, and Lučivjanská (2018) consider informative prior beliefs about return predictability and the effects of this prior information on related economic decisions. I introduce informative priors about market-return predictability, similar to these studies, and investigate the impact on empirical ICAPM tests. Shanken (1987), Harvey and Zhou (1990), Pástor and Stambaugh (1999), (2000), Pástor (2000), and Guidolin and Liu (2016) consider informative priors about the performance of asset pricing models, whereas I specify uninformative priors for cross-sectional test parameters. Finally, Geweke and Zhou (1996) consider uncertainty in latent factors in tests of the arbitrage pricing theory.

The rest of the article is organized as follows: Section II describes the model and estimation procedure. Section III estimates the intertemporal risk factor and tests the ICAPM using uninformative priors. Section IV introduces prior information about market-return dynamics and tests the ICAPM under various informative-prior specifications. Section V concludes.

\section{Methodology}

In this section, I develop an approach for testing the cross-sectional implications of the ICAPM. Section II.A develops a method to estimate the intertemporal risk factor implied by the model. Section II.B presents the cross-sectional test framework. Section II.C outlines estimation, and Section II.D discusses the data.

\section{A. Construction of the Intertemporal Risk Factor}

I test the intertemporal asset pricing model of Campbell (1993), (1996), which provides a discrete-time counterpart to Merton's (1973) ICAPM. Campbell (1993) considers an economy with time-varying expected market returns and Epstein and Zin (1989), (1991) investors. Within this economy, he develops an intertemporal asset pricing equation,

$$
\mathrm{E}_{t} r_{i, t+1}^{e}+\frac{V_{i i}}{2}=\gamma V_{i m}+(\gamma-1) V_{i h}
$$

where lowercase letters denote natural $\operatorname{logs}, r_{i, t+1}^{e}$ is the excess return on stock $i, V_{i i}$ is the variance of stock $i$ 's returns, the $V_{i i} / 2$ term arises from Jensen's inequality with $\log$ returns,

$$
V_{i m}=\operatorname{cov}_{t}\left(r_{i, t+1}^{e}, r_{m, t+1}^{e}\right)
$$


where $r_{m, t+1}^{e}$ is the excess market return, and $V_{i h}$ is a term related to intertemporal risk defined as

$$
V_{i h}=\operatorname{cov}_{t}\left(r_{i, t+1}^{e},\left(\mathrm{E}_{t+1}-\mathrm{E}_{t}\right) \sum_{j=1}^{\infty} \rho^{j} r_{m, t+1+j}\right) .
$$

The $V_{i m}$ term measures exposure to market risk, as in the static CAPM. The $V_{i h}$ term is stock $i$ 's covariance with changes in expected discounted future market returns, which captures the stock's exposure to shifts in the investment opportunity set. $^{2,3}$

Equation (1) implies that changes in expected future market returns can enter as an additional priced risk factor in the cross section. In particular, a representative investor with $\gamma>1$ requires a positive reward for exposure to the intertemporal risk factor, such that stocks that tend to earn poor returns contemporaneous to worsening investment opportunities will have higher expected returns. Shocks to expected market returns are unobservable, however, which is a primary difficulty when testing the ICAPM. Researchers typically approach this problem in one of two ways. In the first test design, several macroeconomic state variables are directly included as additional factors, and the significant pricing of these factors is attributed to the intertemporal hedging motives of the ICAPM. However, ICAPM theory implies that additional state variables should only be priced to the extent that they forecast investment opportunities, and directly including macroeconomic variables exacerbates potential problems of "factor fishing" (Campbell (1996)). The second approach puts economic constraints on ICAPM factors, allowing a factor to be priced only to the extent that it contains information about investment opportunities. I take this approach by directly estimating the intertemporal risk factor implied by the $V_{i h}$ term in equation (1),

$$
f_{h, t+1}=\left(\mathrm{E}_{t+1}-\mathrm{E}_{t}\right) \sum_{j=1}^{\infty} \rho^{j} r_{m, t+1+j}
$$

As Campbell (1996) notes, this approach is less likely to result in spurious factors because any successful factor must tie together the cross-sectional and time-series aspects of the data.

I adopt a Bayesian approach to test the ICAPM for two primary reasons. First, my tests consider the full posterior distribution of the time series of factor realizations $\left\{f_{h, t}\right\}_{t=1}^{T}$ to explicitly account for uncertainty in the intertemporal risk factor, such that the posterior distribution of the price of intertemporal risk reflects this uncertainty. Second, in some specifications, I introduce prior information about investment opportunities using the predictive-system approach of Pástor and Stambaugh (2009). The predictive system allows me to transparently

\footnotetext{
${ }^{2}$ Campbell et al. (2018) develop an ICAPM with stochastic volatility, which produces an additional factor. I consider the Campbell (1993) version of the ICAPM with homoscedastic shocks for analytical tractability.

${ }^{3}$ The $\rho$ parameter in equation (3) is a log-linearization parameter defined by $\rho=1-\exp \left(\mathrm{E}\left(c_{t}-\right.\right.$ $\left.w_{t}\right)$ ), where $c_{t}$ and $w_{t}$ are the logs of consumption and wealth, respectively. Following Campbell (1996), I set $\rho=0.9949$.
} 
introduce prior beliefs about economically meaningful aspects of returns, such as the $R^{2}$ from a predictive regression of market excess returns on the market risk premium.

I use a modified version of Pástor and Stambaugh's (2009) predictive system to estimate the intertemporal risk factor. I first separate the real risk-free rate and the market risk premium for modeling purposes, so equation (4) becomes ${ }^{4}$

$$
f_{h, t+1}=\left(\mathrm{E}_{t+1}-\mathrm{E}_{t}\right) \sum_{j=1}^{\infty} \rho^{j} r_{f, t+1+j}+\left(\mathrm{E}_{t+1}-\mathrm{E}_{t}\right) \sum_{j=1}^{\infty} \rho^{j} r_{m, t+1+j}^{e} .
$$

The changes in expectations of future risk-free rates and market risk premiums are then estimated from the predictive-system vector autoregression (VAR),

$$
\begin{aligned}
r_{m, t}^{e} & =\bar{r}_{m, t-1}^{e}+\eta_{m, t}, \\
r_{n, t} & =r_{f, t}+\bar{\pi}_{t-1}+\eta_{n, t}, \\
\pi_{t} & =\bar{\pi}_{t-1}+\eta_{\pi, t}, \\
x_{t} & =\left(I-\phi_{x}\right) \mathrm{E}_{x}+\phi_{x} x_{t-1}+\eta_{x, t}, \\
\bar{r}_{m, t}^{e} & =\left(1-\phi_{m}\right) \mathrm{E}_{m}+\phi_{m} \bar{r}_{m, t-1}^{e}+\eta_{\bar{m}, t}, \\
r_{f, t+1} & =\left(1-\phi_{r}\right) \mathrm{E}_{r}+\phi_{r} r_{f, t}+\eta_{r, t}, \\
\bar{\pi}_{t} & =\left(1-\phi_{\pi}\right) \mathrm{E}_{\pi}+\phi_{\pi} \bar{\pi}_{t-1}+\eta_{\bar{\pi}, t}, \\
\eta_{t} & \sim \mathrm{N}(0, \Sigma) .
\end{aligned}
$$

Pástor and Stambaugh (2009) estimate a version of this VAR with equations (6a), (6d), (6e), and (6h), and my approach expands the system by incorporating components related to the risk-free rate and inflation. In particular, equations (6a)-(6c) of the VAR describe the dynamics of observed market excess returns, the nominal risk-free rate $\left(r_{n, t}\right)$, and realized inflation $\left(\pi_{t}\right)$. The conditional expectation of market excess returns is the market risk premium, $\bar{r}_{m, t-1}^{e}$. The realized nominal risk-free rate earned in period $t$ is the sum of the real risk-free rate, $r_{f, t}$, and expected inflation, $\bar{\pi}_{t-1}$, plus a measurement error term, and realized inflation is equal to expected inflation plus an error term. ${ }^{5} \mathrm{~A}$ vector of observable state variables $x_{t}$ follows a VAR process given by equation (6d). Equations (6e)-(6g) model latent first-order autoregression (AR(1)) processes for the market risk premium, real risk-free rate, and expected inflation. Finally, equation (6h) specifies that the error terms from equations (6a) $-(6 \mathrm{~g})$ are jointly normally distributed with covariance matrix $\Sigma$.

In contrast to a predictive regression, the predictive system allows for imperfect return prediction. Information from state variables in the predictive system affects the latent-process estimates of the market risk premium, real risk-free rate, and expected inflation through correlated errors from equation (6d) and equations

\footnotetext{
${ }^{4}$ As Cochrane ((2005), p. 9) notes, "Our economic understanding of interest rate variation turns out to have little to do with our understanding of risk premia, so it is convenient to separate the two by looking at interest rates and excess returns separately." Equation (5) closely approximates equation (4) because the monthly risk-free rate is always relatively close to 0 .

${ }^{5}$ The notation for risk-free rates reflects the timing of the period when the rate is earned. Thus, the real risk-free rate denoted by $r_{f, t}$ is earned during period $t$ but known at time $t-1$.
} 
(6e)-(6g). Furthermore, if the latent processes are not perfect linear functions of the state variables, there may be a correlation between the errors in the observations of market excess returns, nominal interest rates, and realized inflation in equations (6a)-(6c) and the errors in equations (6e)-(6g). For instance, as further discussed in Section IV, shocks to current returns and the market risk premium are ex ante likely to be negatively correlated. The predictive system given by equation (6) allows this information to be incorporated into the estimates of the latent processes. $^{6}$

Given the AR(1) structures of $\bar{r}_{m, t}^{e}$ and $r_{f, t+1}$, the change in expectations of future market returns will be a function of the current-period shocks to the market risk premium and the real risk-free rate. The intertemporal risk factor from equation (5) may be written as

$$
f_{h, t+1}=\frac{\eta_{\bar{m}, t+1}}{\phi_{m}\left(1-\phi_{m} \rho\right)}+\frac{\eta_{r, t+1}}{\phi_{r}\left(1-\phi_{r} \rho\right)},
$$

where $\eta_{\bar{m}, t+1}$ and $\phi_{m}$ are defined in equation (6e), $\eta_{r, t+1}$ and $\phi_{r}$ are defined in equation (6f), and $\rho$ is the discounting term from equation (3). ${ }^{7}$ This factor is the change in the infinite sum of expected discounted future market returns given the predictive system in equation (6).

Before proceeding, I note that several alternative methods are available for estimating the intertemporal risk factor. Modeling choices affect the latent-factor estimates, which can, in turn, affect ICAPM inferences. I show in Section C.1 of Appendix $C$ in the Supplementary Material that estimating the intertemporal risk factor with a Bayesian predictive regression produces similar results for the price of intertemporal risk under uninformative priors. I use the predictive system because of its previously noted advantages, but my findings and conclusions about the ICAPM are conditional on the empirical design.

\section{B. A Cross-Sectional Test of the ICAPM}

From equation (1), the ICAPM implies a 2-factor model with a market risk factor and an intertemporal risk factor capturing unexpected changes in expected market returns. I develop a regression-based approach to test the ICAPM's crosssectional implications that has a similar structure to a Fama and MacBeth (1973) methodology. More specifically, I use a hierarchical Bayes method to estimate the system of equations,

$$
\begin{aligned}
r_{i, t, y}^{e} & =\alpha_{i, y}+\beta_{i, y}^{m} r_{m, t, y}^{e}+\beta_{i, y}^{h} f_{h, t, y}+\epsilon_{i, t, y}, \quad \epsilon_{i, t, y} \sim \mathrm{N}\left(0, \sigma_{i, y}^{2}\right), \\
\bar{r}_{i, y}^{e}+\frac{s_{i, y}^{2}}{2} & =\lambda_{0, y}+\lambda_{m, y} \beta_{i, y}^{m}+\lambda_{h, y} \beta_{i, y}^{h}+\epsilon_{i, y}, \quad \epsilon_{i, y} \sim \mathrm{N}\left(0, \sigma_{y}^{2}\right), \\
\lambda_{y} & =\bar{\lambda}+\epsilon_{y}, \quad \epsilon_{y} \sim \mathrm{N}\left(0, V_{\lambda}\right),
\end{aligned}
$$

\footnotetext{
${ }^{6}$ In Section C. 1 of Appendix C in the Supplementary Material, I use a diagnostic tool from Pástor and Stambaugh (2009) to check whether the predictive-regression assumption of an exact linear relation between the expected market return and predictive state variables holds in the data. The results show that returns are imperfectly predicted, which provides additional motivation for adopting the predictive-system approach.

${ }^{7}$ See Appendix A of the Supplementary Material for the derivation.
} 
where $t$ denotes a month within a 3-year period $y ; r_{i, t, y}^{e}$ and $r_{m, t, y}^{e}$ are log real excess returns in month $t$ of period $y$ for asset $i$ and the market, respectively; $f_{h, t, y}$ is the intertemporal risk factor described in the previous section in month $t$ of period $y$; and $\bar{r}_{i, y}^{e}$ and $s_{i, y}^{2}$ are the mean and variance of returns for asset $i$ in period $y$. I test the model using the full cross section of individual stocks.

The system of equations in equations $(8 \mathrm{a})-(8 \mathrm{c})$ measures the prices of risk in the cross section. Factor loadings for each firm $i$ are estimated in equation (8a) during each period $y$, and firm betas are allowed to vary across periods. ${ }^{8}$ Equation (8b) specifies a cross-sectional regression in each period $y$. The $s_{i, y}^{2} / 2$ term is a Jensen's inequality adjustment analogous to the term in the asset pricing equation (1), so the left-hand side of equation (8b) measures the average excess return for stock $i$. Thus, equations (8a) and (8b) measure the relation between factor loadings and average returns in each period. If a particular risk factor is priced in the cross section, exposure to the factor should be systematically rewarded over time. Therefore, I aggregate the price-of-risk estimates from each period to produce the full-period price of risk, $\bar{\lambda}$, in equation (8c). If a component of the vector $\bar{\lambda}$ is different from 0 , there is evidence that the corresponding risk factor is systematically priced in the cross section. In the remainder of the article, I refer to $\bar{\lambda}$ when discussing a price of risk.

\section{Estimation}

I estimate the model using the Markov chain Monte Carlo (MCMC) procedure described in Appendix B of the Supplementary Material. The prior distributions for the predictive system in equation (6) are uninformative for the ICAPM test in Section III.B, and the tests in Section IV incorporate informative priors as described in Section IV.A. Throughout the article, I specify uninformative priors for the hierarchical Bayes parameters in equation (8) in the cross-sectional tests.

For each prior specification, the predictive-system MCMC is run for 500,000 iterations, and the first 100,000 draws are discarded as a burn-in period. To reduce the serial correlation in parameter draws across iterations, I keep every fourth posterior draw of the parameters, such that the remaining 100,000 draws display relatively low serial correlation. The intertemporal risk factor is calculated for each draw using equation (7). Finally, I run the MCMC for the hierarchical Bayes model for 100,000 iterations conditional on the draws from the predictive system. The first 20,000 draws are discarded as a burn-in period, and inferences are based on the remaining 80,000 posterior draws.

\section{Data}

I test the ICAPM over the period Jan. 1952-Dec. 2014. ${ }^{9}$ This 63-year sample period produces 213 -year subperiods in the cross-sectional test. Monthly stock

\footnotetext{
${ }^{8}$ The rolling-window design for estimating betas allows firm risk exposures to flexibly vary across periods, but the method produces an inherent trade-off between using a longer period to more precisely estimate firm factor loadings and allowing loadings to vary more frequently by using shorter windows. Specifying a fixed window length of 3 years appears to provide a good balance given the current setup. Alternative methods avoid fixing a window length and allow for time variation in parameters (e.g., Bianchi, Guidolin, and Ravazzolo (2017)).

${ }^{9}$ Given that the intertemporal risk factor is dependent on a model for the risk-free rate, I choose a starting date for the sample period that follows the Treasury-Federal Reserve Accord of 1951,
} 
return data are from the Center for Research in Security Prices (CRSP), and all ordinary common stocks with share codes of 10 or 11 that are listed on the New York Stock Exchange, American Stock Exchange, or National Association of Securities Dealers Automated Quotations (NASDAQ) are included in the sample. The sample includes a total of 100,908 firm-periods with an average of 4,805 firms per 3-year period. In months in which a firm is delisted, missing stock returns are replaced by the delisting return in CRSP. ${ }^{10}$ To avoid potential survivorship bias to the extent possible, a firm is included in the sample for a given period if it has more than six monthly returns over the 3 -year period. Equation (8a) includes only the months with available returns for firm-periods with incomplete return data.

The market factor in the cross-sectional test is proxied by the value-weighted CRSP stock market index. The predictive system in equation (6) used to estimate the intertemporal risk factor models the market excess return, nominal risk-free rate, and realized inflation. For estimation, I use value-weighted CRSP index excess returns, the 1-month Treasury-bill rate, and personal consumption expenditure (PCE) inflation. I also use four state variables: the term spread (the difference between yields on 10-year and 1-year Treasury bonds), the default spread (the difference between yields on Baa and Aaa bonds as rated by Moody's), the dividend yield (the dividend yield on the CRSP value-weighted index calculated following Cochrane (2008)), and the short-term risk-free rate (the yield on 3-month Treasury bills). ${ }^{11}$ Summary statistics for state variables and returns are shown in Table 1.

\section{ICAPM Tests with Uninformative Priors}

This section tests the ICAPM using uninformative priors for the predictivesystem parameters. Section III.A discusses estimates from the predictive system and reports characteristics of the intertemporal risk factor. Section III.B presents the results from testing the ICAPM.

\section{A. Estimates of the Intertemporal Risk Factor}

The intertemporal risk factor is calculated from unexpected shocks to the market risk premium and real interest rate, as shown in equation (7). To provide intuition on the dynamics of this factor, Figure 1 shows the time series of the market risk premium, real risk-free rate, and expected inflation from the predictive system. The system is estimated using uninformative priors. ${ }^{12}$ Each plot shows the posterior mean and a $90 \%$ credible interval for the variable over the period Jan. 1952-Dec. 2014, and National Bureau of Economic Research (NBER) recessions are shaded.

following Campbell (1996). The Federal Reserve maintained a low-interest-rate peg prior to the accord, so risk-free rates behaved quite differently before the sample period begins.

${ }^{10}$ See Shumway (1997) for a discussion of delisting bias.

${ }^{11}$ All interest rate and inflation data are from the Federal Reserve Bank of St. Louis (http://research .stlouisfed.org/fred2/), and the returns and ex-dividend returns on the value-weighted CRSP index are from CRSP. PCE inflation, excluding food and energy, is seasonally adjusted. I use Consumer Price Index inflation prior to the PCE data start date of Jan. 1959.

${ }^{12}$ Additional information about prior parameters is available in Appendix B of the Supplementary Material. Table C.IV of the Supplementary Material shows posterior means for the predictive-system parameters. 
Table 1 presents summary statistics for predictive-system state variables and returns. Panel A shows the average and standard deviation of the logs of value-weighted Center for Research in Security Prices (CRSP) real stock market portfolio excess returns, the 1-month Treasury bill rate, and personal consumption expenditure (PCE) inflation. Panel B reports summary statistics for the state variables used in the predictive system. The term spread is the difference in yields between 10-year and 1-year Treasury bonds, the default spread is the difference in yields of Baa- and Aaa-rated bonds based on Moody's ratings, the dividend yield is the dividend yield for the value-weighted CRSP stock market index, and the short rate is the yield on 3-month Treasury bills. Panel $\mathrm{C}$ shows the pooled mean and standard deviation of real individual stock excess returns over the sample period and the average number of firms in each period. The means of log variables are reported as the mean plus half of the variance as a Jensen's inequality adjustment. The sample period is Jan. 1952-Dec. 2014

Panel A. Prediction Variable Summary Statistics

\begin{tabular}{lc}
\hline \multicolumn{1}{c}{ Statistic } & $\begin{array}{c}\text { Real Market } \\
\text { Excess Return } \\
r_{m, t}^{e}\end{array}$ \\
Mean & 0.59 \\
Standard deviation & 4.35
\end{tabular}

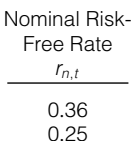

Realized Inflation $\frac{\pi_{t}}{0.25}$ 0.21

Panel B. State Variable Summary Statistics

\begin{tabular}{|c|c|c|c|c|}
\hline Statistic & Term Spread & Default Spread & Dividend Yield & Short Rate \\
\hline Mean & 0.96 & 0.97 & 3.14 & 4.51 \\
\hline Standard deviation & 1.10 & 0.45 & 1.16 & 3.04 \\
\hline \multicolumn{5}{|c|}{ Panel C. Stock Return Summary Statistics } \\
\hline Statistic & & Real Excess Return & & No. of Stocks \\
\hline Mean & & 0.74 & & 4,805 \\
\hline Standard deviation & & 17.79 & & \\
\hline
\end{tabular}

The estimated market risk premium in Graph A of Figure 1 displays substantial time variation. Consistent with theoretical and empirical evidence, the expected excess market return tends to increase during recessions and decline in expansionary periods. Estimates of the risk premium range from over $1 \%$ per month for some of the 1950s and during the recent financial crisis to negative values during the peak periods that precede some recessions in the sample. The market risk premium in any given month is estimated with considerable uncertainty, however; the $90 \%$ credible interval has a range of $1.74 \%$ per month on average.

Graphs B and C of Figure 1 show the real risk-free rate and expected inflation, respectively. The real interest rate varies less than the market risk premium, with the posterior mean ranging from $-0.20 \%$ to $0.71 \%$ per month over the sample period. The series is procyclical, consistent with Ang, Bekaert, and Wei (2008). As anticipated, expected inflation displays spikes in the inflationary periods of the 1970s and 1980s and low, stable inflation in the post-1990 period. Both series are estimated with more precision compared with the market risk premium, and the posterior distributions are tightly distributed around their means. The average ranges of the $90 \%$ credible intervals of the real interest rate and expected inflation are $0.16 \%$ and $0.15 \%$ per month, respectively.

The intertemporal risk factor, $f_{h, t}$, from equation (7) is determined by unexpected changes in the market risk premium and the real interest rate as well as the persistence of each process. The factor therefore inherits the uncertainty in these estimates. Given the relatively diffuse posterior distribution for the market risk premium, I proceed to further analyze uncertainty in the forecasts of the market risk premium. The following analysis of the predictive system with uninformative 


\section{FIGURE 1 \\ Predictive-System Estimates of Latent Processes}

Figure 1 shows estimates of the time series of the market risk premium (Graph A), real interest rate (Graph B), and expected inflation (Graph C) from the predictive system in equation (6) under the base specification with uninformative priors. The solid lines represent the posterior means, and the dotted lines show the $90 \%$ credible intervals for the latent processes. Each variable is expressed in percentages per month. The sample period is Jan. 1952-Dec. 2014, and National Bureau of Economic Research (NBER) recessions are shaded.
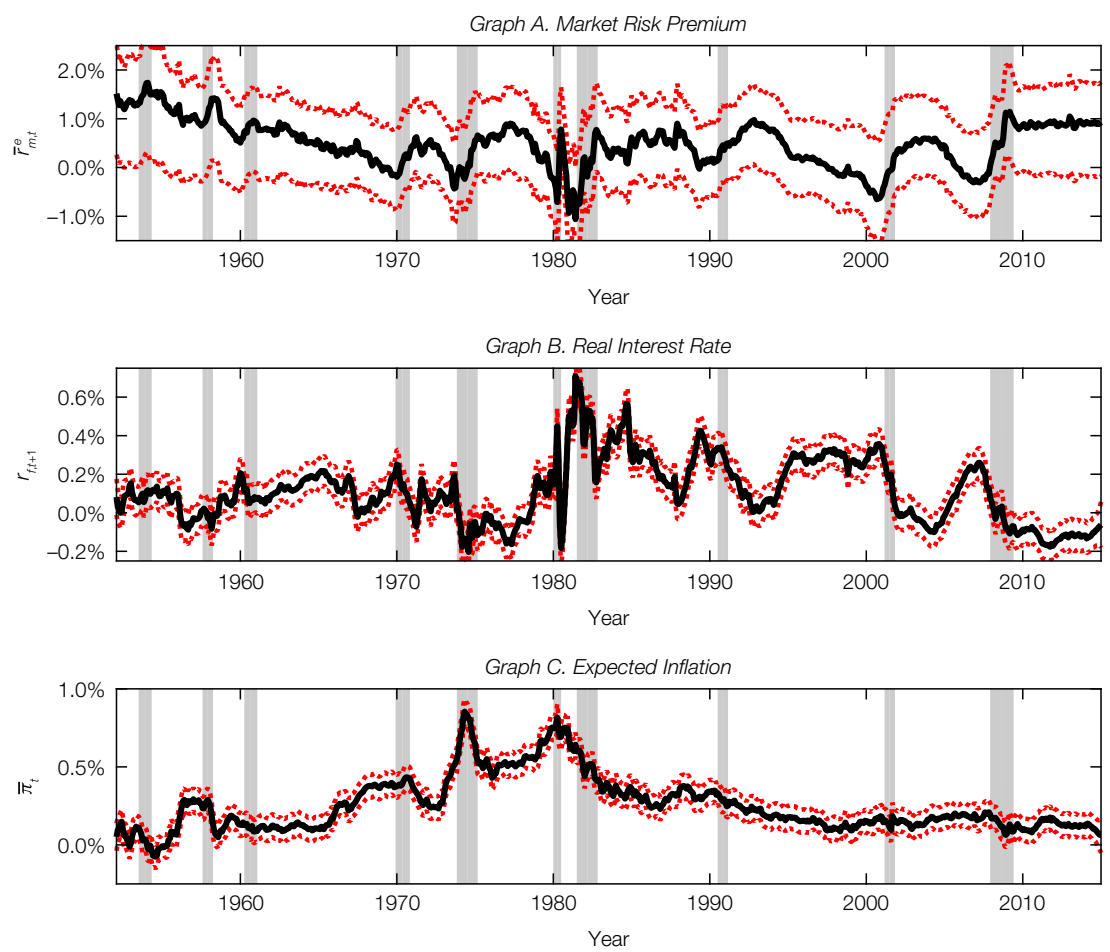

priors also provides a baseline for investigating the specifications with informative priors in Section IV.

Figure 2 shows the posterior distributions of the predictive-regression $R^{2}$ (Graph A) and the correlation between shocks to the current market return and the market risk premium, $\rho_{m \bar{m}}\left(\right.$ Graph B). The $R^{2}$ for each posterior draw is from a predictive regression of monthly market excess returns on the draw of the market risk premium. The $\rho_{m \bar{m}}$ parameter measures the correlation between the errors in equations (6a) and (6e) in each draw.

The predictive system with uninformative priors produces evidence that market returns are predictable. The mean of the predictive $R^{2}$ posterior distribution in Graph A of Figure 2 is approximately $2.2 \%$, which is comparable in magnitude to the $R^{2}$ of $2.6 \%$ obtained from an ordinary least squares (OLS) predictive regression using the term spread, default spread, dividend yield, and short-term interest rate as state variables. The posterior distribution of the predictive $R^{2}$ is relatively diffuse, with a $90 \%$ credible interval spanning from $1.2 \%$ to $3.0 \%$. 
Figure 2 shows posterior distributions for the predictive-regression $R^{2}$ (Graph A) and the $\rho_{m \bar{m}}$ parameter (Graph B) from the predictive system in equation (6) under uninformative priors. The $R^{2}$ in each posterior draw is from a predictive regression of monthly excess market returns on the time series of the market risk premium for that draw. The $\rho_{m \bar{m}}$ parameter measures the correlation between shocks to current market returns and the market risk premium. The sample period is Jan. 1952-Dec. 2014.
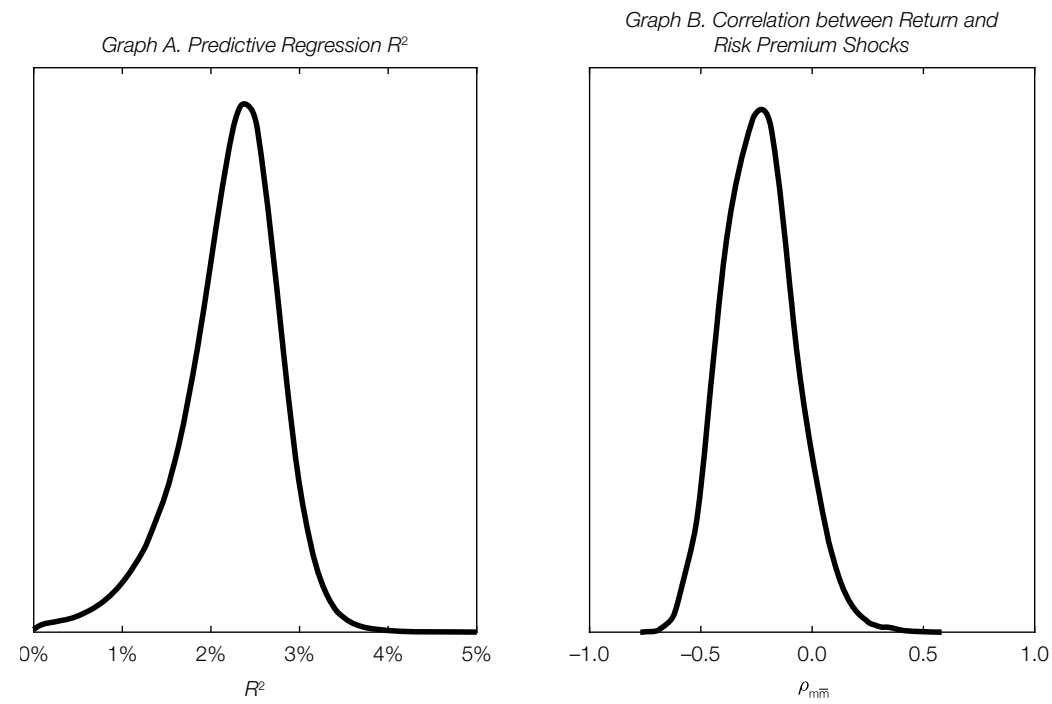

The posterior distribution of $\rho_{m \bar{m}}$ in Graph B of Figure 2 suggests that the correlation between shocks to market returns and the market risk premium is negative. The posterior mean of the correlation is -0.24 , and most of the posterior distribution lies below 0 . Economically, a negative value of $\rho_{m \bar{m}}$ is consistent with a valuation framework in which shocks to future discount rates produce a portion of current return variance, as suggested by Campbell (1991) and others.

The predictive system also derives information from the state variables when forecasting market returns. As shown by Pástor and Stambaugh (2009), the effects of the state variables can be translated into predictive-regression slopes of market excess returns on the variables. Figure 3 graphs the posterior distributions of the implied regression slopes for the variables of term spread, default spread, dividend yield, and short rate. The term spread and dividend yield are positive return predictors with posterior means of 0.15 (90\% credible interval of $0.00-0.33)$ and 0.24 (90\% credible interval of $0.06-0.48)$, respectively. The short rate is a negative predictor with a posterior mean of -0.12 ( $90 \%$ credible interval of -0.24 to $-0.03)$. Finally, the default spread is the least reliable predictor, with a positive posterior mean and a $90 \%$ credible interval of -0.14 to 0.30 . Information from the term spread and short rate may particularly be important for ICAPM inferences because these state variables have predictive content for future market returns, but their innovations exhibit relatively low correlation with the market factor. In contrast, dividend-yield shocks are highly negatively correlated with current market returns, such that the information about investment opportunities contained in this variable is aligned with market-factor realizations. 


\section{FIGURE 3 \\ Posteriors of Implied Predictive-Regression Slopes for State Variables}

Figure 3 shows posterior distributions for the predictive-regression slopes implied by the predictive system in equation (6) under uninformative priors. The predictive-regression slopes are calculated from the predictive-system parameters following Pástor and Stambaugh (2009). The slopes measure the relation between the market risk premium and the term spread (Graph A), default spread (Graph B), dividend yield (Graph C), and short rate (Graph D). The sample period is Jan. 1952-Dec. 2014.
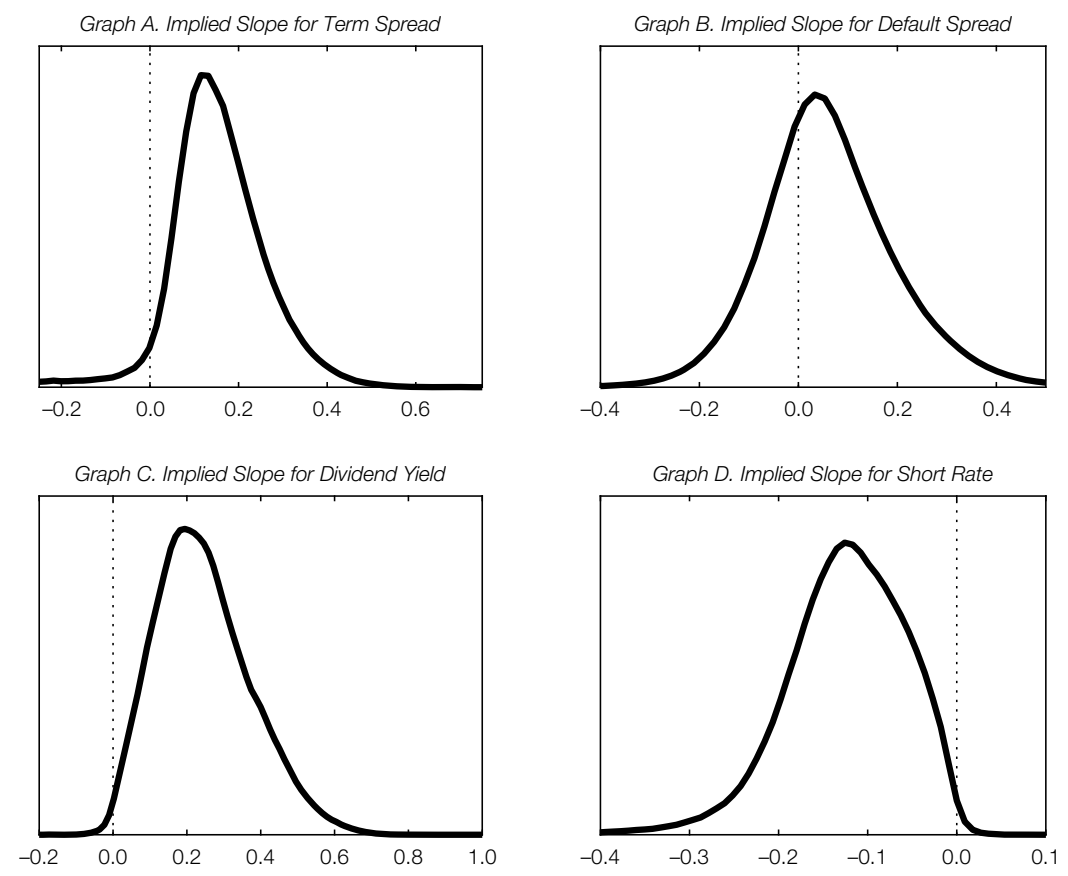

Table 2 shows time-series summary statistics for the intertemporal risk factor, which is calculated as the shock to the infinite sum of discounted future expected returns following equation (7). I specifically report the posterior average of the time-series mean, standard deviation, and correlation with the market factor. The $90 \%$ credible intervals for these statistics are in square brackets. The factor has an average mean of $0.00 \%$ and an average time-series standard deviation of $4.39 \%$. This level of variation is similar in magnitude to that of the market factor, which has a standard deviation of $4.35 \%$, as shown in Table 1 . The $90 \%$ credible interval of the standard deviation ranges from $1.67 \%$ to $9.04 \%$, such that the volatility of the intertemporal risk factor varies substantially across draws. The intertemporal risk factor is likely negatively correlated with the market factor, as expected. The degree of correlation is modest, however, with an average correlation of -0.23 across the posterior draws and a $90 \%$ credible interval of -0.48 to 0.05 . Low correlation indicates that the intertemporal risk factor may contain important incremental information relative to the market factor. Finally, Table 2 reports the time-series average of the posterior standard deviation of the intertemporal risk factor. In an average month, the standard deviation of the posterior distribution 
Table 2 presents statistics for the intertemporal risk factor, which is estimated based on the predictive system in equation (6) with uninformative priors. The mean, standard deviation, and correlation with the market factor reported for the intertemporal risk factor are calculated as averages of these time-series statistics across posterior draws, and the numbers in square brackets show the $90 \%$ credible interval for the posterior distribution of each statistic. The average factor uncertainty is the time-series mean of the posterior standard deviation of the monthly intertemporal risk factor. The mean and standard deviations are reported in percentages per month. The sample period is Jan. 1952-Dec. 2014.

\begin{tabular}{lc} 
Statistic & $\begin{array}{c}\text { Intertemporal } \\
\text { Risk Factor }\end{array}$ \\
\cline { 1 - 2 } Mean & 0.00 \\
Standard deviation & {$[-0.29,0.28]$} \\
Correlation with market factor & 4.39 \\
Average factor uncertainty & {$[-0.67,9.04]$} \\
& 3.0 .23 \\
\hline
\end{tabular}

of the factor realization for the month is $3.84 \%$, indicating that substantial uncertainty exists about the additional factor in the ICAPM.

\section{B. ICAPM Test Results}

Table 3 shows the estimated prices of risk for the factor models. The reported estimates are means of the posterior distributions, and posterior $90 \%$ credible intervals are in square brackets. Consistent with theoretical predictions, estimates for the CAPM indicate that market risk is positively rewarded, with a posterior mean of $0.75 \%$ per month and a $90 \%$ credible interval of $0.22 \%-1.29 \%$, and the model intercept is centered at $-0.01 \%$. This finding of a substantial positive price of risk for market exposure among individual stocks is in line with research by Davies (2010) and Lewellen (2015). ${ }^{13}$ The root-mean-square error (RMSE), which measures the pricing errors in 3-year average returns of the model expressed in percentages per month, is $2.28 \%$ on average for the CAPM. The model thus achieves some gains relative to pricing errors assuming equal expected returns across all stocks; the RMSE without considering beta is $3.40 \%$ per month.

Table 3 also reports results for the ICAPM. The market factor continues to carry a positive price-of-risk estimate of $0.63 \%$ per month with a $90 \%$ credible interval of $0.25 \%-1.01 \%$. The posterior mean of the intercept is $0.20 \%$, and the $90 \%$ credible interval includes 0 . The price of risk for the intertemporal risk factor, $\bar{\lambda}_{h}$, has a positive posterior mean at $0.52 \%$. However, the posterior standard deviation of this estimate is quite large $(0.44 \%)$, such that the $90 \%$ credible interval for $\bar{\lambda}_{h}$ is $-0.11 \%$ to $1.30 \%$ per month. Despite the relatively low degree of

\footnotetext{
${ }^{13}$ Davies (2010) shows that the hierarchical Bayes approach mitigates the errors-in-variables (EIV) problem relative to the standard Fama-MacBeth (1973) technique. Using the Fama-MacBeth approach, there is still support for a positive price of market risk among individual stocks. Using the full sample of firms, a Fama-MacBeth test relating returns to contemporaneous betas produces estimates of an intercept of $0.53 \%$ (standard error of $0.20 \%$ ) and a price of risk of $0.18 \%$ (standard error of $0.09 \%$ ). This sample includes firms with partial return data during the period, which may exacerbate the EIV bias due to the inclusion of betas that are imprecisely estimated from a few data points. Conditioning on firms with no missing returns during each 3-year period introduces a potential survivorship bias but reduces the EIV bias, and resulting estimates show an intercept of $0.50 \%$ (standard error of $0.18 \%$ ) and price of risk of $0.38 \%$ (standard error of $0.13 \%$ ).
} 
TABLE 3

Estimated Prices of Risk for ICAPM Factors under Uninformative Priors

Table 3 reports the prices of risk for the intertemporal capital asset pricing model (ICAPM) market and intertemporal risk factors. The intertemporal risk factor is estimated based on the predictive system in equation (6) with uninformative priors. The table shows estimates for the prices of risk from the system of equations in equations (8a)-(8c), along with the root-mean-square error (RMSE). The capital asset pricing model (CAPM) is included for comparison. The numbers in square brackets show the $90 \%$ credible interval for the posterior distribution of each parameter. The sample period is Jan. 1952-Dec. 2014.

\begin{tabular}{|c|c|c|c|c|}
\hline \multirow[b]{2}{*}{ Model } & \multicolumn{2}{|c|}{$\mathrm{E}_{t} r_{i}^{e}+\frac{V_{i i}}{2}=\bar{\lambda}_{0}+\bar{\lambda}_{m} \beta_{i}^{m}+\bar{\lambda}_{h} \beta_{i}^{h}$} & \multirow[b]{2}{*}{$\bar{\lambda}_{h}$} & \multirow[b]{2}{*}{ RMSE } \\
\hline & $\bar{\lambda}_{0}$ & $\bar{\lambda}_{m}$ & & \\
\hline CAPM & $\begin{array}{c}-0.008 \\
{[-0.583,0.566]}\end{array}$ & $\begin{array}{c}0.753 \\
{[0.217,1.286]}\end{array}$ & & $\begin{array}{c}2.280 \\
{[2.279,2.282]}\end{array}$ \\
\hline ICAPM under uninformative priors & $\begin{array}{c}0.203 \\
{[-0.199,0.597]}\end{array}$ & $\begin{array}{c}0.628 \\
{[0.254,1.006]}\end{array}$ & $\begin{array}{c}0.515 \\
{[-0.112,1.302]}\end{array}$ & $\begin{array}{c}1.918 \\
{[1.726,2.168]}\end{array}$ \\
\hline
\end{tabular}

evidence that intertemporal risk is priced, the ICAPM tends to produce gains in model fit relative to the CAPM, with an average RMSE of $1.92 \% .{ }^{14}$

Overall, the results in this section provide mixed evidence about the ICAPM. The intertemporal risk factor is estimated with considerable uncertainty, and this uncertainty is inherited by the posterior for the price of intertemporal risk. Although the posterior mean of $\bar{\lambda}_{h}$ is positive, the $90 \%$ credible interval includes 0 . Hence, the ICAPM specification with uninformative priors produces only weak evidence that intertemporal risk is priced in the cross section of stocks.

\section{ICAPM Tests with Prior Information about Investment Opportunities}

This section tests the ICAPM with economically motivated priors on aspects of market-return predictability. Incorporating prior information will influence the estimated intertemporal risk factor and may affect inferences about the pricing of intertemporal risk. Section IV.A develops informative priors for predictive-system parameters and analyzes prior predictions relative to historical information. Section IV.B presents empirical results from cross-sectional ICAPM tests across prior specifications.

\section{A. Informative Priors in the Predictive System}

\section{Prior Parameters}

I specify informative priors for parameters that govern the market risk premium in the predictive system. The predictive system has prior parameters for the

\footnotetext{
${ }^{14}$ In Section C. 1 of Appendix C in the Supplementary Material, I estimate the intertemporal risk factor using a predictive-regression approach rather than the predictive system. The intertemporal risk factor derived from the predictive regression is apparently estimated with much less uncertainty; the standard deviation of the factor posterior draws averages $1.98 \%$ across months. Imposing the constraint that the market risk premium is an exact linear function of state variables in the predictive regression makes it easier to learn about the market risk premium (Pástor and Stambaugh (2012)), and this assumption of the predictive regression is violated in the data, as shown in Section C.1 of Appendix C in the Supplementary Material. Nevertheless, the ICAPM inferences using the predictiveregression approach are similar to the base case; the posterior for the price of intertemporal risk has a positive mean but a $90 \%$ credible interval that includes 0 .
} 
long-run expectation $\left(\mathrm{E}_{m}\right)$ and persistence parameter $\left(\phi_{m}\right)$ from equation (6e) as well as the relevant variance and covariance elements of $\Sigma$ in equation (6h). Pástor and Stambaugh (2009) develop methods to conveniently express covariancematrix priors for the predictive system in terms of economically meaningful quantities. In particular, the prior distribution for the relevant elements of $\Sigma$ is determined by setting parameters that correspond to priors about i) the $R^{2}$ from a predictive regression of market excess returns on the market risk premium and ii) the correlation between shocks to current returns and the market risk premium in equations (6a) and (6e), $\rho_{m \bar{m}}$.

I primarily concentrate on informative priors for $R^{2}$ and $\rho_{m \bar{m}}$ because these aspects of the dynamics of the market risk premium are most important for ICAPM inferences. I form a 2-dimensional grid over the prior parameters for $R^{2}$ and $\rho_{m \bar{m}}$. In the predictive $R^{2}$ dimension, I consider a range of $0 \%-3 \%$ for a monthly predictive-regression $R^{2}{ }^{15}$ The prior contains information equivalent to a pseudosample with $T / 2$ monthly observations, where $T$ is the number of months in my empirical sample. In the second dimension, the prior distribution of $\rho_{m \bar{m}}$ is approximately uniform in each specification but with different bounds across cases. Specifically, the lower bound of the $\rho_{m \bar{m}}$ prior remains at -0.9 , and the upper bound takes values between -0.9 and 0.9 across grid points, such that the prior mean of $\rho_{m \bar{m}}$ ranges from -0.9 to 0.0 across the prior-parameter values. Concentrating on the space of priors with negative means of $\rho_{m \bar{m}}$ is motivated by the Campbell (1991) decomposition as well as empirical evidence from LeRoy and Porter (1981), Shiller (1981), Campbell and Ammer (1993), Van Binsbergen and Koijen (2010), and others that market returns tend to be negatively related to discount-rate shocks. I specify 13 equally spaced grid points in each dimension to generate 169 prior-parameter combinations.

ICAPM inferences are much less sensitive to the prior distributions for the $\mathrm{E}_{m}$ and $\phi_{m}$ parameters in equation (6e). To facilitate the prior predictive analysis introduced in Section IV.A.2, I specify a somewhat informative prior for the persistence parameter, such that $\phi_{m} \in[0,1]$ is normally distributed, with a mean of 0.97 and a relatively large standard deviation of 0.50. I show in Appendix C of the Supplementary Material that the ICAPM inferences are very similar for a specification with uninformative priors on $\phi_{m}$. Finally, the long-run expectation $\left(\mathrm{E}_{m}\right)$ is normally distributed, with a mean equal to the sample mean market excess return and a large standard deviation of 10. It is important to note that I continue to specify uninformative priors for the cross-sectional test parameters in the system of equations in equations ( $8 \mathrm{a})-(8 \mathrm{c})$, such that prior information is only introduced within the predictive system to estimate the intertemporal risk factor for use in the ICAPM test.

\footnotetext{
${ }^{15} \mathrm{~A}$ range of $0 \%-3 \%$ for the monthly predictive-regression $R^{2}$ prior parameter encompasses most in-sample-regression $R^{2}$ figures from previous studies, which are often around $1 \%$. The prior parameter pertains to the $R^{2}$ from a hypothetical monthly predictive regression on the market risk premium, which is likely to exceed the typical $R^{2}$ figures from empirical studies that condition on specific state variables to model the variation in expected returns.
} 


\section{Prior Predictive Analysis}

Perspective on the region of the prior-parameter space that is reasonable can be gained using a prior predictive analysis with variance ratios. Following Poterba and Summers (1988), the $k$-period variance ratio is defined as

$$
\operatorname{VR}(k)=\frac{\operatorname{Var}\left(r_{m, t \rightarrow t+k}^{e}\right)}{k \operatorname{Var}\left(r_{m, t}^{e}\right)},
$$

where $r_{m, t \rightarrow t+k}^{e}$ is the $k$-period cumulative log excess market return. A variance ratio of 1 indicates that variance grows linearly with the horizon, which is the prediction from independent and identically distributed (IID) models. Within a predictive system, variance ratios depend on $R^{2}$ and $\rho_{m \bar{m}}$. Specifically, I show in Appendix A of the Supplementary Material that the $k$-period variance ratio can be expressed as

$$
\operatorname{VR}(k)=1+A(k)\left(R^{2}\right)^{1 / 2}\left(1-R^{2}\right)^{1 / 2} \rho_{m \bar{m}}+B(k) R^{2},
$$

where $A(k)$ and $B(k)$ are positive constants that increase with the horizon $k$ when the equity premium is persistent (i.e., $\phi_{m}>0$ ). If $\rho_{m \bar{m}}<0$ as expected, the last two terms of the variance ratio in equation (10) have opposite signs. The first of these terms captures the effect of mean reversion, which has a negative effect on multiperiod variance ratios. Mean reversion has a stronger negative effect on the variance ratio when shocks to current and future returns are highly negatively correlated (i.e., $\rho_{m \bar{m}}$ is negative and large) and when expected returns vary substantially over time (i.e., $R^{2}$ is high). The second term shows the additional variance in cumulative multiperiod returns that is generated through time variation in the persistent expected return. This term is large when the market risk premium is highly variable (i.e., $R^{2}$ is high). In sum, the $R^{2}$ and $\rho_{m \bar{m}}$ parameters that are important for ICAPM inferences interact to determine variance ratios.

An advantage of using variance ratios to investigate the prior-parameter space is that historical variance ratios can be calculated using annual return data and do not require data on any additional state variables. As such, prior beliefs about $R^{2}$ and $\rho_{m \bar{m}}$ can be formed using estimated historical variance ratios from a long sample period that predates my ICAPM testing period. In particular, I use annual data on real stock market returns from Jeremy Siegel that span the period 1802-1951, giving a 150-year time series of returns that could have been used to form prior beliefs at the beginning of the sample period. Table 4 reports historical variance-ratio estimates for horizons of 2-8 years, following Poterba and Summers (1988). Consistent with past literature, the estimated variance ratios are below 1 and decrease in horizon to reach 0.58 at an 8 -year horizon, which is indicative of relatively strong mean-reversion effects in historical stock returns.

Given a set of prior-parameter values for $R^{2}$ and $\rho_{m \bar{m}}$, I use a prior predictive analysis to consider whether the implied prior beliefs are consistent with the variance ratios from historical data. Specifically, I draw 100,000 time series of 1,800 monthly stock market excess returns from their prior distribution given a set of prior parameters and the structure of the predictive system in equation (6). I then compound the monthly stock returns to produce 150-year samples of annual stock returns and calculate variance ratios for horizons of $2-8$ years. This process 
Table 4 shows variance ratios for horizons of 2-8 years using annual real log stock market returns. Variance ratios are defined as the variance of $k$-year cumulative returns divided by $k$ times the variance of 1 -year returns. The sample period is $1802-1951$.

\begin{tabular}{lc}
\hline Horizon & Variance Ratio \\
\cline { 2 - 2 } 2 years & 0.990 \\
3 years & 0.859 \\
4 years & 0.801 \\
5 years & 0.738 \\
6 years & 0.635 \\
7 years & 0.603 \\
8 years & 0.576 \\
\hline
\end{tabular}

produces the implied prior distributions for variance ratios at each horizon length for each set of prior parameters. Finally, I examine whether the observed historical variance ratios lie within the $90 \%$ credible intervals across the 2 - to 8 -year horizons.

Figure 4 shows the region of the prior-parameter space that produces prior beliefs about variance ratios that are consistent with pre-1952 data. The shaded area denotes prior-parameter combinations for which the historical variance ratios lie within the $90 \%$ credible intervals. ${ }^{16}$ In general, prior specifications with more predictable returns and a relatively strong negative correlation between shocks to current returns and the market risk premium are most consistent with observed variance ratios. This finding is in line with equation (10) because these parameter combinations will generate substantial mean-reversion effects to produce prior distributions for variance ratios that are centered well below 1. Based on the prior predictive analysis, the shaded region in Figure 4 represents the portion of the prior-parameter space that is most reasonable given historical return information.

\section{Estimates of the Intertemporal Risk Factor under Informative Priors}

I estimate the predictive system in equation (6) for each combination of $R^{2}$ and $\rho_{m \bar{m}}$ priors to produce draws for the intertemporal risk factor. Figure 5 summarizes the posterior distributions of the predictive-regression $R^{2}$ (Graph A) and $\rho_{m \bar{m}}$ (Graph B) from the predictive systems across prior specifications. The 7 subplots report results for different $R^{2}$ priors ranging from $0 \%$ to $3 \%$, and each subplot shows the posteriors associated with 7 prior parameters for $\rho_{m \bar{m}}$ that produce prior means ranging from -0.9 to 0.0 . The 49 cases displayed in the figure are a subset of the 169 prior-parameter combinations from the grid developed in Section IV.A.1. For each prior-parameter combination, the box shows the median and the 25th and 75th percentiles of the posterior distribution, and the whiskers encompass the $90 \%$ credible interval. The $R^{2}=0.0 \%$ prior produces a degenerate prior at $0 \%$ for the standard deviation of shocks to the market risk premium, such that $\rho_{m \bar{m}}$ is not well defined, and Graph B is left empty for this case.

\footnotetext{
${ }^{16}$ Figure 4 shows results from interpolating across the grid points based on the percentage of draws in which the historical variance ratios lie within the $90 \%$ credible interval. Appendix C of the Supplementary Material contains a version of Figure 4 without interpolation that indicates whether the variance ratios lie within the $90 \%$ credible intervals at each of the 169 grid points.
} 
FIGURE 4

Region of the Prior-Parameter Space That Is Consistent with Historical Variance Ratios

Figure 4 shows the region of the prior-parameter space in which the historical variance ratios at horizons of 2-8 years lie within the $90 \%$ credible interval of the variance ratios from a prior predictive analysis. The prior-parameter space is a 2-dimensional grid over prior parameters corresponding to the predictive-regression $R^{2}$ and the correlation between shocks to current returns and the market risk premium, $\rho_{m \bar{m}}$. Historical variance ratios are calculated using annual data on real stock market returns from 1802-1951.

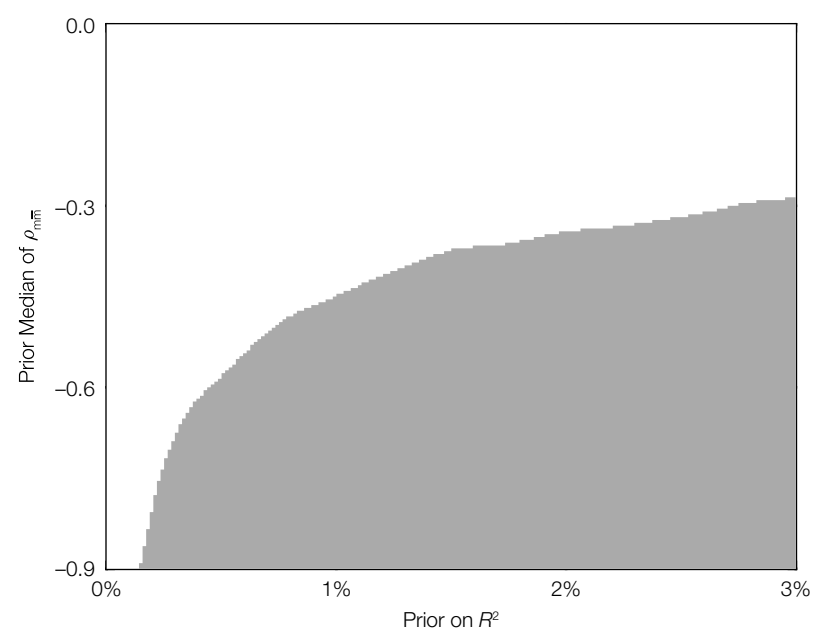

Three general patterns emerge from the posterior distributions of $R^{2}$ and $\rho_{m \bar{m}}$ in Figure 5. First, priors associated with low levels of return predictability (i.e., $R^{2}=0.5 \%$ or $1.0 \%$ ) produce posteriors of predictive $R^{2}$ that are shifted upward relative to the prior distributions and relatively similar across $\rho_{m \bar{m}}$ priors, with posterior means ranging from $0.9 \%$ to $1.0 \%$ for $R^{2}=0.5 \%$ specifications and $1.6 \%-1.7 \%$ with $R^{2}=1.0 \%$ priors. Across these priors, the posteriors of $\rho_{m \bar{m}}$ indicate a relatively large negative correlation between shocks to current returns and the market risk premium; the posterior means range from -0.80 to -0.60 in these cases. Second, specifications with prior $R^{2}=1.5 \%-3.0 \%$ and a large negative prior mean of -0.90 for $\rho_{m \bar{m}}$ produce posteriors that are distinctive relative to cases with looser priors on $\rho_{m \bar{m}}$. The predictive $R^{2}$ posteriors are quite diffuse and centered near their priors, and the posterior distributions of $\rho_{m \bar{m}}$ are highly concentrated, with posterior means ranging from -0.89 to -0.84 across prior $R^{2}$ parameters.

The third pattern in Figure 5 is that the group of specifications with $R^{2}$ priors from $1.5 \%$ to $3.0 \%$ and $\rho_{m \bar{m}}$ prior means ranging from -0.6 to 0.0 produce relatively similar posterior distributions. Specifically, these cases produce evidence of substantial return predictability, with $R^{2}$ posterior means between $2.4 \%$ and $2.8 \%$. The posteriors also indicate relatively low negative correlations between shocks to current returns and the market risk premium, with posterior means of $\rho_{m \bar{m}}$ ranging from -0.42 to -0.31 . Overall, this set of specifications shows the most similarity to the results for uninformative priors in Section III in terms of the magnitudes of $R^{2}$ and $\rho_{m \bar{m}}$, but the posteriors for the informative-prior cases in Figure 5 are noticeably tighter compared with the corresponding posteriors in Figure 2. 
FIGURE 5

Posteriors of Important Features of Market-Return Predictability across Prior Specifications

Figure 5 shows quantiles of the posterior distributions of the $R^{2}$ from a predictive regression of market excess returns on the market risk premium (Graph A) and the correlation between shocks to current returns and the market risk premium, $\rho_{m \bar{m}}$ (Graph B), across specifications of the predictive-system prior parameters for the predictive-regression $R^{2}$ and the correlation between shocks to current returns and the market risk premium. The box shows the median and 25th and 75 th percentiles, and the whiskers encompass the $90 \%$ credible interval. The intertemporal risk factor is estimated with the predictive system in equation (6), with informative priors as described in Section IV.A. The predictive-regression $R^{2}$ is based on monthly returns. The sample period is Jan. 1952-Dec. 2014.
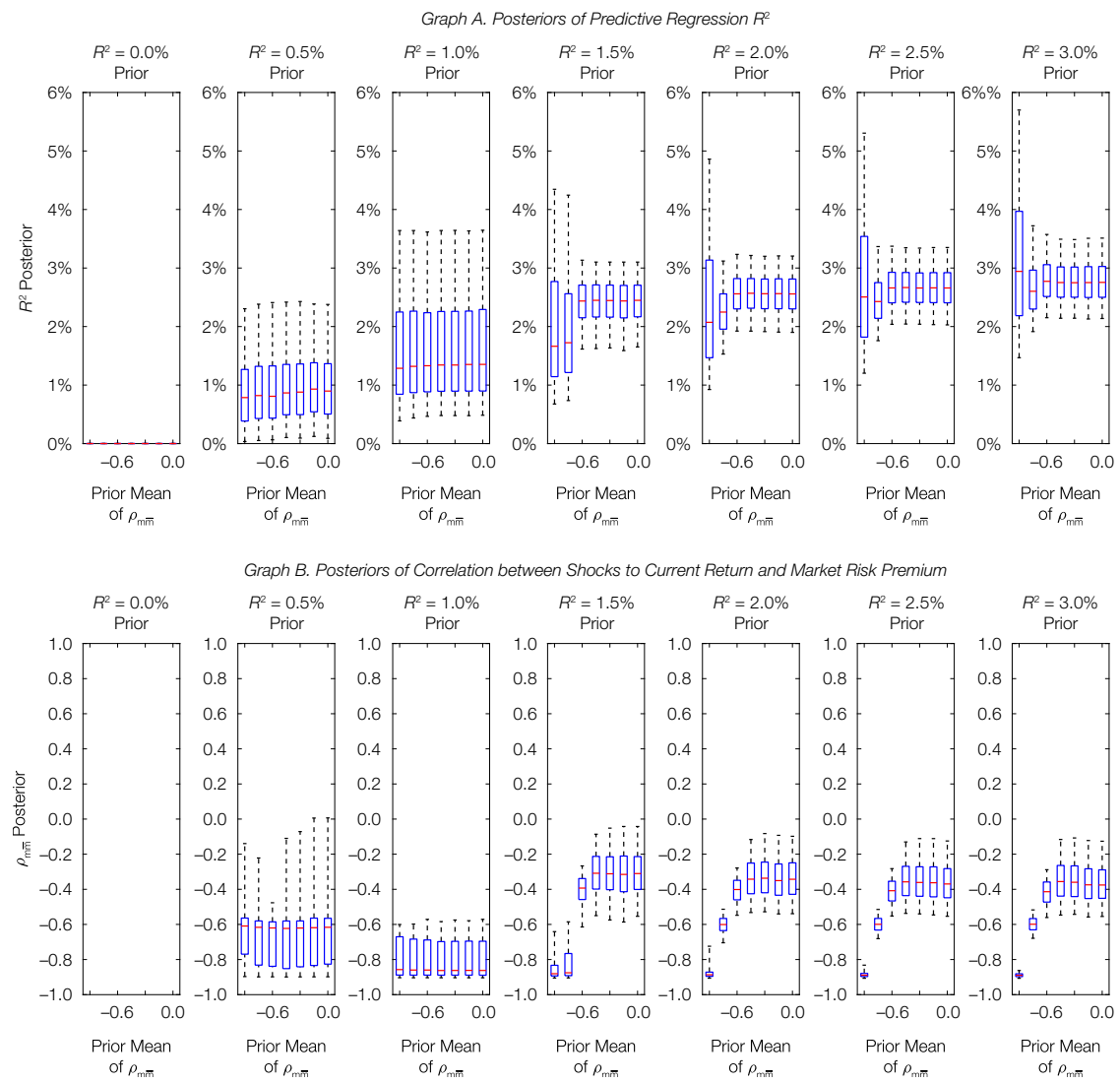

Graph B. Posteriors of Correlation between Shocks to Current Return and Market Risk Premium
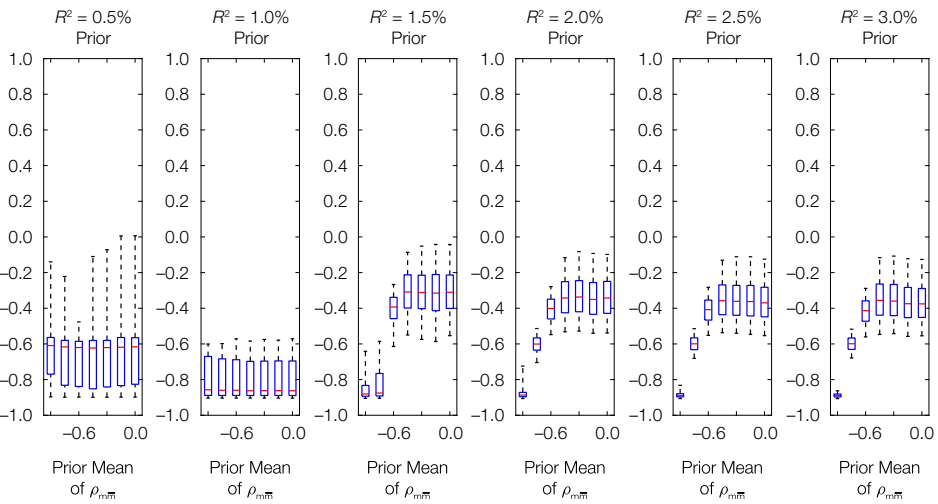

Figure 6 shows the posteriors of the implied predictive-regression slopes for the state variables across prior specifications. The dividend yield is an important predictor in all cases, with the magnitude of its relation to returns increasing in the prior $R^{2}$ parameter. The term-spread and short-rate state variables, conversely, are primarily important for the group of priors with $R^{2}=1.5 \%-3.0 \%$ and a prior mean of $\rho_{m \bar{m}}$ from -0.6 to 0.0 . The role of these state variables for this set of prior specifications is again relatively similar to that of the uninformative case, with posteriors that are similarly located but more concentrated compared with those in Figure 3. As previously discussed, the abilities of the term-spread and short-rate variables to predict returns are potentially important for producing an intertemporal risk factor with information that is orthogonal to the market factor. 


\section{FIGURE 6}

\section{Posteriors of Implied Predictive-Regression Slopes for State Variables across Prior Specifications}

Figure 6 shows quantiles of the posterior distributions for the predictive-regression slopes implied by the predictive system in equation (6) across specifications of the prior parameters for the predictive-regression $R^{2}$ and the correlation between shocks to current returns and the market risk premium. The predictive-regression slopes are calculated from the predictive-system parameters following Pástor and Stambaugh (2009). The box shows the median and 25th and 75th percentiles, and the whiskers encompass the $90 \%$ credible interval. The slopes measure the relation between the market risk premium and the term spread (Graph A), default spread (Graph B), dividend yield (Graph C), and short rate (Graph D). The sample period is Jan. 1952-Dec. 2014.
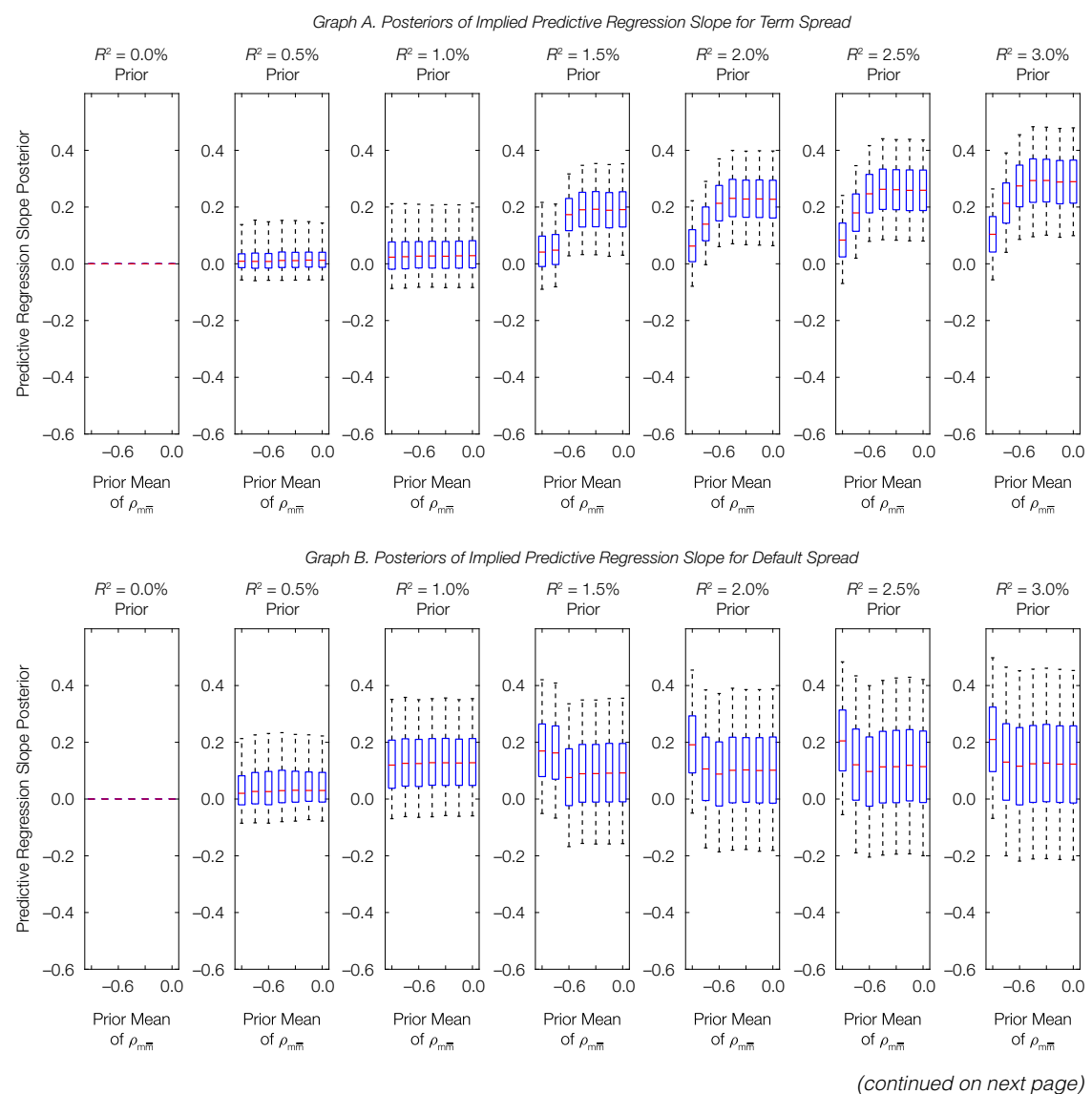

The posterior for the intertemporal risk factor is calculated using equation (7) for each prior specification. The characteristics of the intertemporal risk factor are influenced by the $R^{2}$ and $\rho_{m \bar{m}}$ features of the market risk premium as well as the relation between the risk-free-rate and market-risk-premium components of the factor. In particular, the market-risk-premium component will be particularly important when $R^{2}$ is high because strong predictability implies that the market risk premium is highly variable. Furthermore, large negative values of $\rho_{m \bar{m}}$ indicate that the market-risk-premium component of the intertemporal risk factor will be closely related to the market factor, whereas lower degrees of correlation imply that the intertemporal risk factor contains more orthogonal information. Finally, 
FIGURE 6 (continued)

Posteriors of Implied Predictive-Regression Slopes for State Variables across Prior Specifications
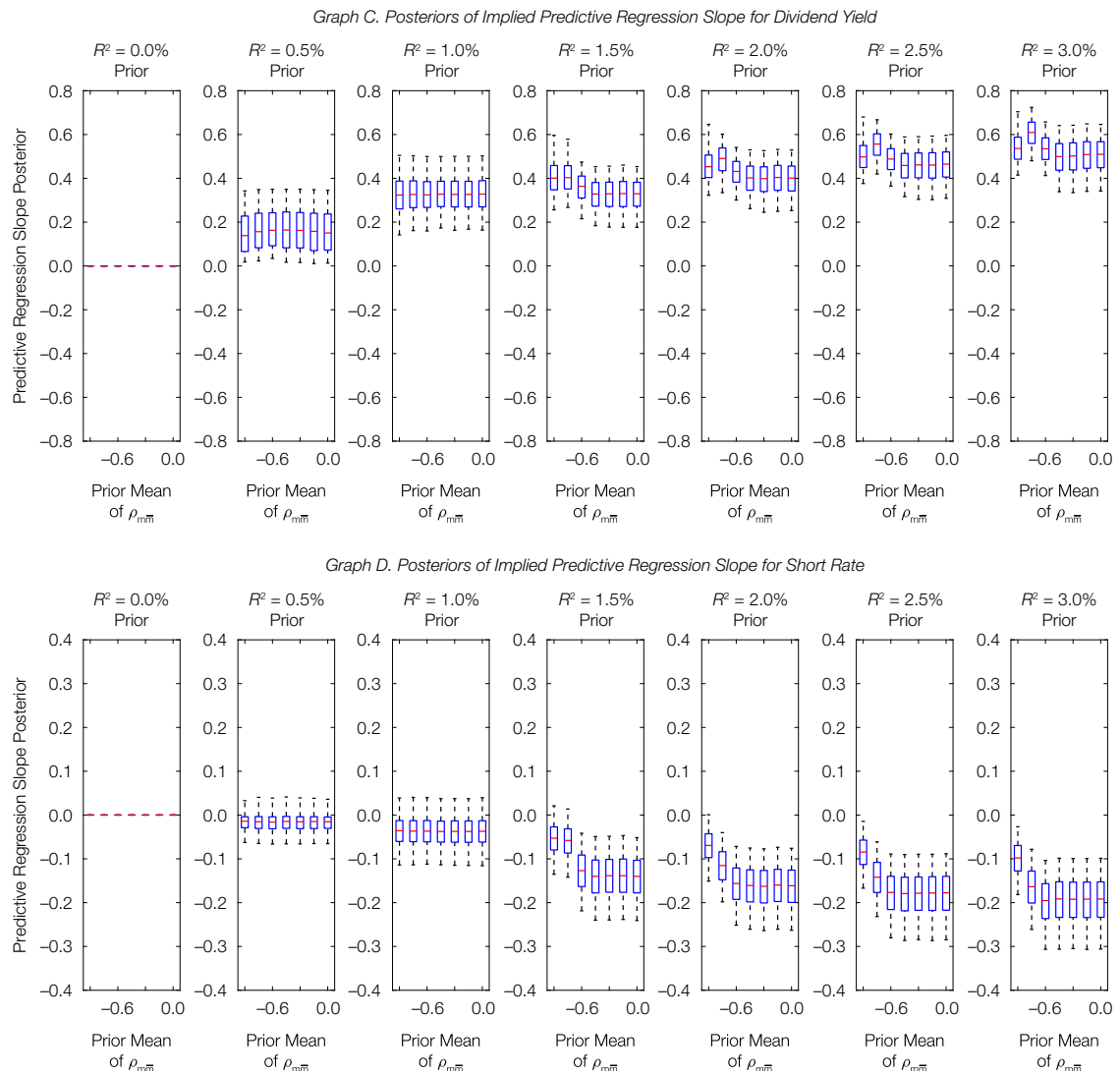

the relation between the risk-free-rate and market-risk-premium components is important. For example, if an improvement in economic conditions in a given month tends to produce an increase in the expected real risk-free rate and a decrease in the market risk premium, then the two components will partially offset when they are combined to calculate the realization of the intertemporal risk factor for that month. In contrast, combining risk-free-rate and market-risk-premium components that are unrelated will produce a more volatile intertemporal risk factor.

Figure 7 displays the posteriors of time-series statistics for the estimated intertemporal risk factor associated with each set of priors. Graph A shows the posteriors of the time-series standard deviation, and Graph B focuses on the correlation between the two risk factors in the ICAPM. ${ }^{17}$ For the $R^{2}=0.0 \%$ cases,

\footnotetext{
${ }^{17}$ Figures corresponding to the posterior of the mean of the intertemporal risk factor and the timeseries average for the posterior standard deviation of draws of the intertemporal risk factor are available in Section C.1 of Appendix C in the Supplementary Material.
} 


\section{FIGURE 7}

Posteriors of Statistics for the Intertemporal Risk Factor across Prior Specifications

Figure 7 shows quantiles of the posterior distributions of time-series statistics for the intertemporal risk factor across specifications of the predictive-system prior parameters for the predictive-regression $R^{2}$ and the correlation between shocks to current returns and the market risk premium. Graph A displays the posteriors of the time-series standard deviation of the intertemporal risk factor, $f_{h, t}$. Graph B shows the posteriors of the correlation between the market factor and the intertemporal risk factor. The box shows the median and 25th and 75th percentiles, and the whiskers encompass the $90 \%$ credible interval. The intertemporal risk factor is estimated with the predictive system in equation (6) with informative priors as described in Section IV.A. The standard deviation of the intertemporal risk factor is expressed in percentages per month. The sample period is Jan. 1952-Dec. 2014.

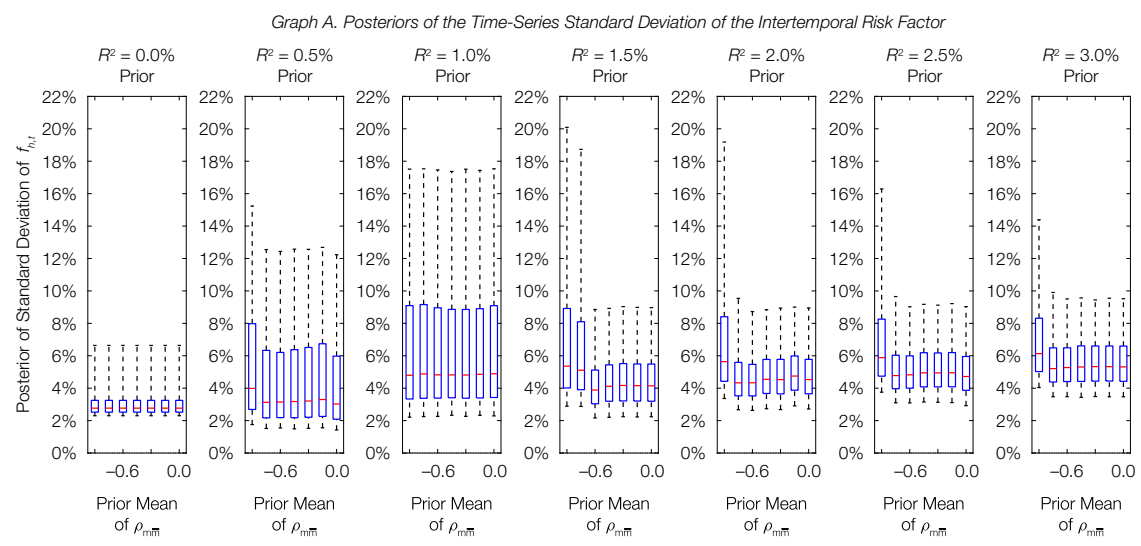

Graph B. Posteriors of Correlation between Market and Intertemporal Risk Factors
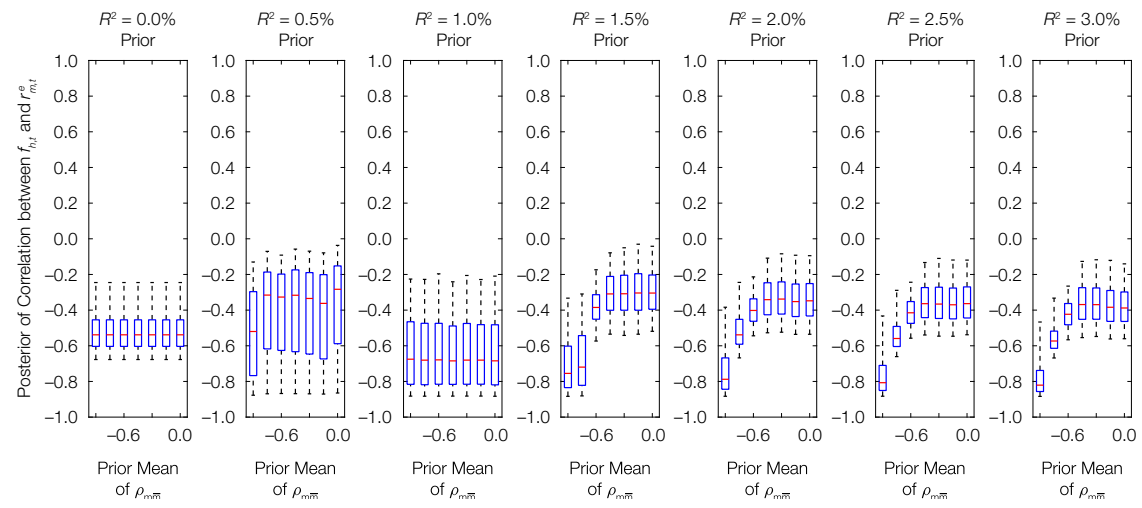

there is no variation in the market risk premium, such that the intertemporal risk factor is determined entirely by variation in expected risk-free rates. The standard deviation of the intertemporal risk factor has a posterior mean of $3.26 \%(90 \%$ credible interval of $2.30 \%-6.64 \%$ ), and the posterior mean of the correlation between the risk factors is $-0.54(90 \%$ credible interval of -0.68 to -0.25$)$ in this case.

The posteriors of the intertemporal risk factor show varying patterns across the remaining prior-parameter space, as shown in Figure 7. Given priors of relatively low market-return predictability (i.e., $R^{2}$ priors of $0.5 \%$ or $1.0 \%$ ), the degree of variation in the market risk premium is fairly low and uncertain (consistent with the $R^{2}$ posteriors in Graph A of Figure 5). Furthermore, the risk-free-rate and 
market-risk-premium components of the intertemporal risk factor are nearly uncorrelated for these prior specifications, and the risk-free-rate component is particularly important with lower levels of market-return predictability. These features combine to generate the relatively diffuse posteriors of the intertemporal risk factor's standard deviation and correlation with the market factor that are shown in Figure 7.

Figure 7 shows that the posteriors in the prior-parameter space with higher degrees of return predictability (i.e., prior $R^{2}$ of $1.5 \%$ or higher) have interesting patterns. In particular, the characteristics of the intertemporal risk factor are quite different for the prior specifications with a prior mean of -0.9 for $\rho_{m \bar{m}}$ compared with the models with prior means of -0.6 to 0.0 . The intertemporal risk factor is highly volatile and strongly negatively correlated with the market factor across the $\rho_{m \bar{m}}=-0.9$ prior specifications, with the average factor standard deviation ranging from $7.33 \%$ to $7.44 \%$ and the average correlation with the market factor ranging between -0.77 and -0.70 across the $R^{2}=1.5 \%-3.0 \%$ cases. The risk-free-rate and market-risk-premium components show little correlation in these models, and the relatively strong variation in the market risk premium implies that the intertemporal risk factor is largely dominated by the variation in the market risk premium. In contrast, the average factor standard deviations for the $\rho_{m \bar{m}}=-0.6$ to 0.0 cases are much lower at $4.45 \%-5.85 \%$, and these prior specifications also produce intertemporal risk factors that are less negatively correlated with the market factor, with average correlations of -0.42 to -0.30 . An important feature of this prior-parameter space is that shocks to the risk-free-rate and market-risk-premium components have an average correlation of -0.42 to -0.36 , which is consistent with real rates increasing simultaneously with a declining market risk premium. The two shocks thus tend to partially offset and modulate the intertemporal risk factor.

Finally, the lower correlation between the market factor and the intertemporal risk factor implies a greater role for cash-flow risk for the set of priors with $\rho_{m \bar{m}}=-0.6$ to 0.0 compared with the $\rho_{m \bar{m}}=-0.9$ priors. I confirm in Section C. 1 of Appendix C in the Supplementary Material that the discount-rate risk dominates for the models with priors of a strong negative correlation between shocks to current returns and the market risk premium, whereas the cash-flow risk is quite important for the weaker-correlation cases. Large negative values of the $\rho_{m \bar{m}}$ prior parameter encourage the predictive system to concentrate on information in state variables with innovations that are strongly correlated with current market returns, consistent with the dependence of the $\rho_{m \bar{m}}=-0.9$ priors on the dividend-yield state variable in Figure 6. With looser priors on $\rho_{m \bar{m}}$, however, the predictive system allows for stronger roles from the term-spread and short-rate variables that may contain additional information about macroeconomic conditions but have shocks that are less closely related to realized returns.

\section{B. Cross-Sectional Test Results}

Figure 8 shows the results from testing the ICAPM in the cross section of stocks given each set of prior parameters. Specifically, the figure plots the posterior distributions of the intercept (Graph A), the price of risk for the market factor (Graph B), and the price of risk for the intertemporal risk factor (Graph C). The 


\section{FIGURE 8 \\ Posteriors of Prices of Risk for ICAPM Factors across Prior Specifications}

Figure 8 shows quantiles of the posterior distributions of the intercept $\left(\bar{\lambda}_{0}\right)$ in Graph $A$, the price of risk for the market factor $\left(\bar{\lambda}_{m}\right)$ in Graph B, and the price of risk for the intertemporal risk factor $\left(\bar{\lambda}_{h}\right)$ in Graph C across specifications of the predictive-system prior parameters for the predictive-regression $R^{2}$ and the correlation between shocks to current returns and the market risk premium. The box shows the median and 25 th and 75 th percentiles, and the whiskers encompass the $90 \%$ credible interval. The intertemporal risk factor is estimated with the predictive system in equation (6), with informative priors as described in Section IV.A. The intercept and prices of risk for intertemporal capital asset pricing model (ICAPM) factors are expressed in percentages per month. The sample period is Jan. 1952-Dec. 2014.

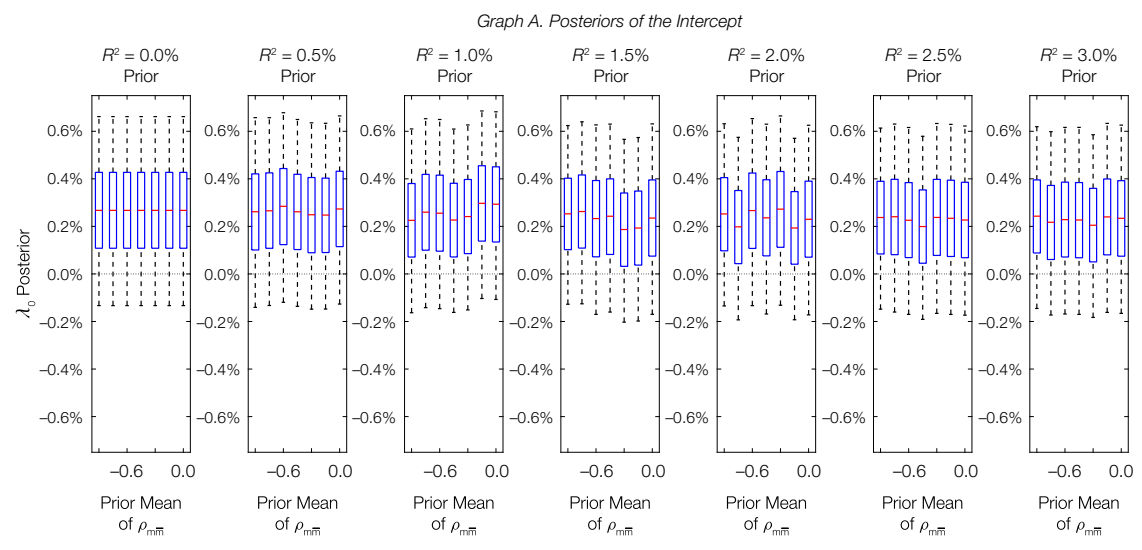

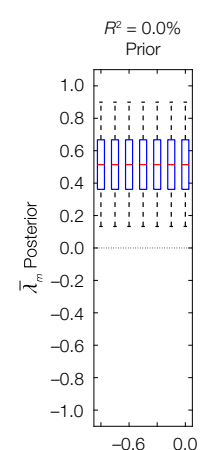

Prior Mean of $\rho_{\mathrm{m} \bar{m}}$

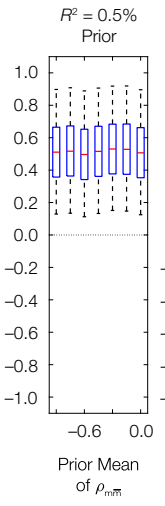

of $\rho_{\operatorname{mim}}$

Graph B. Posteriors of the Price of Market Risk
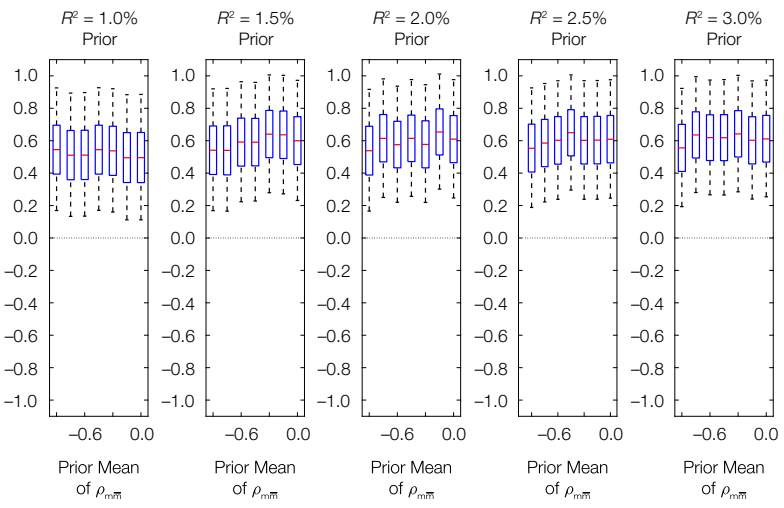

(continued on next page)

posterior draws come from estimating the system of equations in equations (8a)(8c) given the posterior of the intertemporal risk factor in each case. Graphs A and $\mathrm{B}$ show that the posteriors of the intercept and the price of market risk are similar across prior specifications, with the $90 \%$ credible intervals of the intercept containing 0 and the posteriors for the price of market risk lying almost entirely above 0 in all cases. These parameter estimates are thus in line with the ICAPM predictions for each prior specification.

The posteriors in Graph $\mathrm{C}$ of Figure 8 indicate that the intertemporal risk factor is positively priced for several sets of prior parameters. Multiple priorparameter combinations with relatively high market-return predictability along with loose priors on $\rho_{m \bar{m}}$ produce large, positive posterior means of $\bar{\lambda}_{h}$ and $90 \%$ 
FIGURE 8 (continued)

Posteriors of Prices of Risk for ICAPM Factors across Prior Specifications

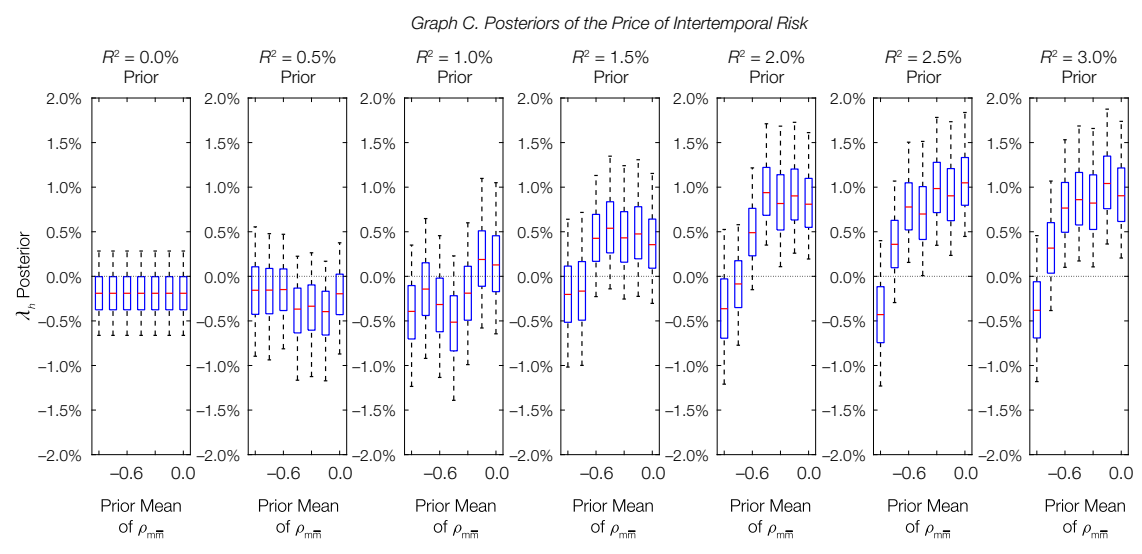

credible intervals that do not contain 0. Specifically, these conditions hold for all sets of priors in Figure 8 with $R^{2}=2.0 \%-3.0 \%$ and $\rho_{m \bar{m}}$ prior means from -0.45 to 0.00 as well as the $\rho_{m \bar{m}}=-0.60$ prior with $R^{2}=2.5 \%$ or $3.0 \%$. Across these cases, the posterior mean of $\bar{\lambda}_{h}$ ranges from $0.78 \%$ to $1.08 \%$ per month, such that the estimated intertemporal risk factor carries a large, positive price of risk in support of ICAPM predictions under these prior specifications. The strong evidence from these cross-sectional tests of an additional priced risk factor in the cross section of stocks also constitutes a rejection of the CAPM.

There is little evidence that the estimated intertemporal risk factor is priced in the remaining cases in Figure 8. The $R^{2}=0.0 \%$ priors produce posteriors of $\bar{\lambda}_{h}$ that are centered at $-0.19 \%$ per month $(90 \%$ credible interval of -0.66 to 0.28 ). Given that the intertemporal risk factor is equivalent to the risk-free-rate component in this case, this result indicates that exposure to unexpected shocks to the real risk-free rate is not priced in the cross section. The other cases with priors of relatively low market-return predictability (i.e., prior $R^{2}$ values of $0.5 \%$ or $1.0 \%$ ) similarly produce little evidence that intertemporal risk is priced; the $90 \%$ credible interval for each posterior distribution of $\bar{\lambda}_{h}$ contains 0 in each case. Finally, the priors with relatively high return predictability of $R^{2}=2.0 \%-3.0 \%$ along with a prior $\rho_{m \bar{m}}$ parameter of -0.9 produce negative posterior means of $\bar{\lambda}_{h}$ ranging from $-0.42 \%$ to $-0.36 \%$. The $90 \%$ credible intervals each contain 0 , such that there is little evidence that the intertemporal risk factor is priced under these prior specifications.

Based on the analysis of the intertemporal risk factors in Section IV.A.3, the group of prior specifications for which intertemporal risk is strongly positively priced corresponds to estimated intertemporal risk factors that share important characteristics. The intertemporal risk factors in these specifications are relatively less volatile and have weaker negative correlations with the market factor compared with the sets of priors for which intertemporal risk is unpriced. Furthermore, cash-flow risk plays a particularly important role in these cases relative to the specifications in which intertemporal risk is not priced. These priors produce 
posteriors of the predictive-system parameters and the time series of the intertemporal risk factor that most closely resemble the posteriors with uninformative priors from Section III, but the informative priors produce posteriors with relatively more precision compared with the uninformative-prior case. The ICAPM test results indicate that the additional information from the priors is helpful in establishing that intertemporal risk is positively priced in accord with model predictions.

The results in Figure 8 show the existence of an additional priced risk factor, such that the single-factor CAPM is rejected. Under several prior specifications, the priced risk factor corresponds to the intertemporal risk factor from Campbell's (1993) ICAPM, such that it ties together the time-series and crosssectional aspects of returns. As a final step in the analysis, I consider these results in the context of the prior predictive analysis in Section IV.A.2. In particular, I investigate whether there is a region of the prior-parameter space that is ex ante reasonable given the variance-ratio analysis and simultaneously produces positive results for the ICAPM.

Figure 9 shows the regions of the prior-parameter space that are consistent with historical variance ratios (light shading), the $90 \%$ credible interval of the price of intertemporal risk that does not include 0 (medium shading), and the overlapping region (dark shading). Figure 9 shows that there is a relatively large region of ex ante reasonable prior parameters that produce evidence that intertemporal risk is priced in the cross section of stocks. The overlapping region comprises prior-parameter values for $R^{2}$ in excess of $1.5 \%$ and prior means of $\rho_{m \bar{m}}$ that range from -0.7 to -0.3 . These prior-parameter combinations are simultaneously

\section{FIGURE 9}

Region of the Prior-Parameter Space in Which Intertemporal Risk Is Priced

Figure 9 shows the region of the prior-parameter space in which 0 is not in the $90 \%$ credible interval of the price of risk for the intertemporal risk factor in dark gray, the region from Figure 4 in which the historical variance ratios at horizons of 2-8 years lie within the $90 \%$ credible interval of the variance ratios from a prior predictive analysis in light gray, and the overlapping region in black. The prior-parameter space is a 2-dimensional grid over prior parameters corresponding to the predictive-regression $R^{2}$ and the correlation between shocks to current returns and the market risk premium, $\rho_{m \bar{m}}$. Historical variance ratios are calculated using annual data on real stock market returns from 1802-1951, and the sample period for intertemporal capital asset pricing model (ICAPM) tests is Jan. 1952-Dec. 2014.

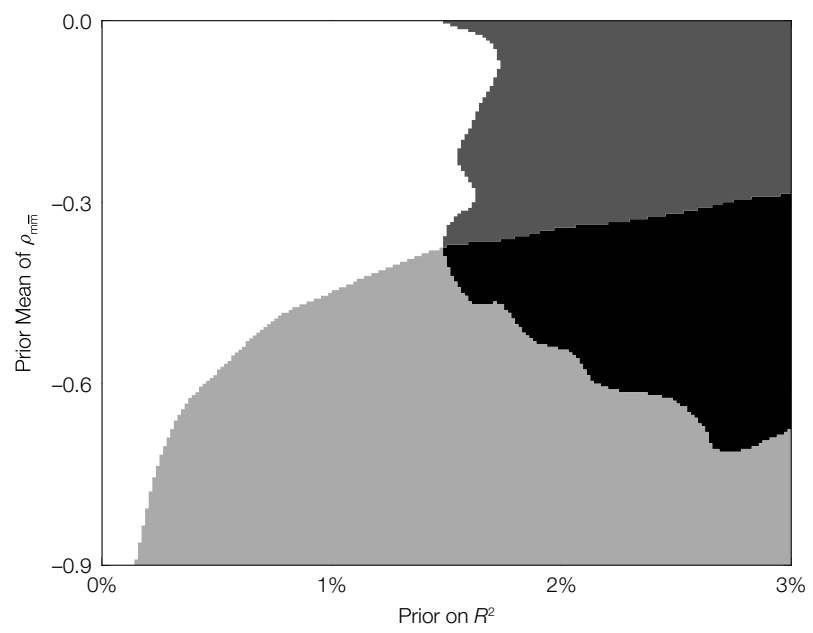


capable of i) producing long-horizon variance ratios that match the low estimates from historical data and ii) generating intertemporal risk factors in which the market risk premium plays an important role but is not forced to be strongly negatively related to market returns. Overall, the results in this section indicate that there is a relatively large region of the prior-parameter space in which the cross-sectional implications of the ICAPM are supported.

\section{Conclusion}

The ICAPM of Campbell (1993) predicts that exposure to unexpected changes in expected future market returns may be priced in the cross section of stocks. The fact that changes in expected market returns are unobservable, however, makes testing the cross-sectional implications of the model more difficult. I introduce a Bayesian approach to test the ICAPM that accounts for uncertainty in the intertemporal risk factor and allows for economically motivated priors on the relation of market-return predictability.

Whereas there is only weak evidence that intertemporal risk is priced under uninformative priors, I find that ICAPM predictions are strongly supported within a relatively large region of an informative prior-parameter space. In particular, the intertemporal risk factor carries a large, positive price-of-risk estimate for prior specifications with substantial market-return predictability and loose priors on the correlation between shocks to current returns and the market risk premium. The estimated intertemporal risk factors in these cases are characterized by a strong role of cash-flow risk in market-return variance and a relatively low correlation with the market factor. Much of this prior-parameter space overlaps with a region that is deemed to be ex ante reasonable given historical stock market variance ratios from the presample period, indicating support for the cross-sectional implications of the ICAPM.

\section{Supplementary Material}

Supplementary Material for this article is available at https://doi.org/10.1017/ S0022109018000972.

\section{References}

Ang, A.; G. Bekaert; and M. Wei. "The Term Structure of Real Rates and Expected Inflation.” Journal of Finance, 63 (2008), 797-849.

Avramov, D. "Stock Return Predictability and Model Uncertainty." Journal of Financial Economics, 64 (2002), 423-458.

Avramov, D. "Stock Return Predictability and Asset Pricing Models." Review of Financial Studies, 17 (2004), 699-738.

Avramov, D.; S. Cederburg; and K. Lučivjanská. "Are Stocks Riskier over the Long Run? Taking Cues from Economic Theory." Review of Financial Studies, 31 (2018), 556-594.

Avramov, D., and T. Chordia. "Asset Pricing Models and Financial Market Anomalies." Review of Financial Studies, 19 (2006), 1001-1040.

Barberis, N. "Investing for the Long Run When Returns Are Predictable." Journal of Finance, 55 (2000), 225-264.

Bianchi, D.; M. Guidolin; and F. Ravazzolo. "Macroeconomic Factors Strike Back: A Bayesian Change-Point Model of Time-Varying Risk Exposures and Premia in the U.S. Cross-Section." Journal of Business and Economic Statistics, 35 (2017), 110-129. 
Boons, M. "State Variables, Macroeconomic Activity, and the Cross Section of Individual Stocks." Journal of Financial Economics, 119 (2016), 489-511.

Brennan, M. J.; A. W. Wang; and Y. Xia. "Estimation and Test of a Simple Model of Intertemporal Capital Asset Pricing.” Journal of Finance, 59 (2004), 1743-1775.

Campbell, J. Y. "A Variance Decomposition for Stock Returns.” Economic Journal, 101 (1991), 157-179.

Campbell, J. Y. "Intertemporal Asset Pricing without Consumption Data." American Economic Review, 83 (1993), 487-512.

Campbell, J. Y. “Understanding Risk and Return.” Journal of Political Economy, 104 (1996), 298-345.

Campbell, J. Y., and J. Ammer. "What Moves the Stock and Bond Markets? A Variance Decomposition for Long-Term Asset Returns." Journal of Finance, 48 (1993), 3-37.

Campbell, J. Y.; S. Giglio; and C. Polk. "Hard Times." Review of Asset Pricing Studies, 3 (2013), 95-132.

Campbell, J. Y.; S. Giglio; C. Polk; and R. Turley. "An Intertemporal CAPM with Stochastic Volatility." Journal of Financial Economics, 128 (2018), 207-233.

Campbell, J. Y.; C. Polk; and T. Vuolteenaho. "Growth or Glamour? Fundamentals and Systematic Risk in Stock Returns.” Review of Financial Studies, 23 (2010), 305-344.

Campbell, J. Y., and T. Vuolteenaho. "Bad Beta, Good Beta." American Economic Review, 94 (2004), 1249-1275.

Cochrane, J. H. Asset Pricing. Princeton, NJ: Princeton University Press (2005).

Cochrane, J. H. "The Dog That Did Not Bark: A Defense of Return Predictability." Review of Financial Studies, 21 (2008), 1533-1575.

Cremers, K. J. M. “Stock Return Predictability: A Bayesian Model Selection Perspective.” Review of Financial Studies, 15 (2002), 1223-1249.

Davies, P. "A Cross-Sectional Test of the CAPM at the Firm Level.” Working Paper, Rutgers University (2010).

Epstein, L. G., and S. E. Zin. "Substitution, Risk Aversion, and the Temporal Behavior of Consumption and Asset Returns: A Theoretical Framework." Econometrica, 57 (1989), 937-969.

Epstein, L. G., and S. E. Zin. "Substitution, Risk Aversion, and the Temporal Behavior of Consumption and Asset Returns: An Empirical Analysis.” Journal of Political Economy, 99 (1991), 263-286.

Fama, E. F. "Efficient Capital Markets: II.” Journal of Finance, 46 (1991), 1575-1617.

Fama, E. F., and J. MacBeth. "Risk, Return, and Equilibrium: Empirical Tests." Journal of Political Economy, 81 (1973), 607-636.

Geweke, J., and G. Zhou. "Measuring the Pricing Error of the Arbitrage Pricing Theory." Review of Financial Studies, 9 (1996), 557-587.

Guidolin, M., and H. Liu. "Ambiguity Aversion and Underdiversification." Journal of Financial and Quantitative Analysis, 51 (2016), 1297-1323.

Harvey, C. R., and G. Zhou. "Bayesian Inference in Asset Pricing Tests.” Journal of Financial Economics, 26 (1990), 221-254.

Hodrick, R. J.; D. T. Ng; and P. Sengmueller. "An International Dynamic Asset Pricing Model." International Tax and Public Finance, 6 (1999), 597-620.

Kandel, S., and R. F. Stambaugh. "On the Predictability of Stock Returns: An Asset Allocation Perspective.” Journal of Finance, 51 (1996), 385-424.

LeRoy, S. F., and R. D. Porter. "The Present-Value Relation: Tests Based on Implied Variance Bounds." Econometrica, 49 (1981), 555-574.

Lewellen, J. "The Cross-Section of Expected Stock Returns.” Critical Finance Review, 4 (2015), 1-44.

Maio, P. "Intertemporal CAPM with Conditioning Variables." Management Science, 59 (2013), $122-141$.

Merton, R. C. “An Intertemporal Capital Asset Pricing Model.” Econometrica, 41 (1973), 867-887.

Pástor, Ľ. "Portfolio Selection and Asset Pricing Models." Journal of Finance, 55 (2000), 179-223.

Pástor, Ľ., and R. F. Stambaugh. "Costs of Equity Capital and Model Mispricing." Journal of Finance, 54 (1999), 67-121.

Pástor, L., and R. F. Stambaugh. "Comparing Asset Pricing Models: An Investment Perspective." Journal of Financial Economics, 56 (2000), 335-381.

Pástor, Ľ., and R. F. Stambaugh. "Predictive Systems: Living with Imperfect Predictors.” Journal of Finance, 64 (2009), 1583-1628.

Pástor, Ľ., and R. F. Stambaugh. "Are Stocks Really Less Volatile in the Long Run?” Journal of Finance, 67 (2012), 431-478.

Pettenuzzo, D.; A. Timmermann; and R. Valkanov. "Forecasting Stock Returns under Economic Constraints." Journal of Financial Economics, 114 (2014), 517-553.

Poterba, J. M., and L. H. Summers. "Mean Reversion in Stock Prices." Journal of Financial Economics, 22 (1988), 27-59. 
Shanken, J. "A Bayesian Approach to Testing Portfolio Efficiency.” Journal of Financial Economics, 19 (1987), 195-215.

Shiller, R. J. "Do Stock Prices Move Too Much to Be Justified by Subsequent Changes in Dividends?" American Economic Review, 71 (1981), 421-436.

Shumway, T. "The Delisting Bias in CRSP Data.” Journal of Finance, 52 (1997), 327-340.

Van Binsbergen, J. H., and R. S. J. Koijen. "Predictive Regressions: A Present-Value Approach." Journal of Finance, 65 (2010), 1439-1471.

Wachter, J. A., and M. Warusawitharana. "Predictable Returns and Asset Allocation: Should a Skeptical Investor Time the Market?" Journal of Econometrics, 148 (2009), 162-178.

Wachter, J. A., and M. Warusawitharana. "What Is the Chance That the Equity Premium Varies over Time? Evidence from Regressions on the Dividend-Price Ratio." Journal of Econometrics, 186 (2015), 74-93. 